\title{
Ensemble Properties of Comets in the Sloan Digital Sky Survey
}

\author{
Michael Solontoi $^{\mathrm{a}}$, Željko Ivezić ${ }^{\mathrm{b}}$, Mario Jurićc,d \\ Andrew C. Becker ${ }^{b}$, Lynne Jones ${ }^{\mathrm{b}}$, Andrew A. West ${ }^{\mathrm{f}}$, \\ Steve Kent ${ }^{\mathrm{g}}$ Robert H. Lupton ${ }^{\mathrm{e}}$, Mark Claire ${ }^{\mathrm{b}}$, \\ Gillian R. Knapp ${ }^{\mathrm{e}}$, Tom Quinn ${ }^{\mathrm{b}}$, James E. Gunn ${ }^{\mathrm{e}}$, \\ Donald P. Schneider ${ }^{h}$ \\ a Adler Planetarium, 1300 S. Lake Shore Drive, Chicago, IL 60605, USA \\ ${ }^{\mathrm{b}}$ University of Washington, Dept. of Astronomy, Box 351580, Seattle, WA 98195, \\ USA \\ ${ }^{\mathrm{c}}$ Hubble Fellow \\ ${ }^{\mathrm{d}}$ Harvard College Observatory, Cambridge, MA 02138, USA \\ e Princeton University Observatory, Princeton, NJ 08544, USA \\ ${ }^{\mathrm{f}}$ Department of Astronomy, Boston University, 725 Commonwealth Ave, Boston, \\ MA 02215, USA \\ ${ }^{\mathrm{g}}$ Fermi National Accelerator Laboratory, Batavia, IL 60510, USA \\ ${ }^{\mathrm{h}}$ Department of Astronomy and Astrophysics, Pennsylvania State University, \\ University Park, PA 16802, USA
}

Number of pages: 20

Number of tables: 8

Number of figures: 11

Preprint submitted to Icarus

11 May 2018 
Proposed Running Head:

Ensemble Properties of Comets in the Sloan Digital Sky Survey

Please send Editorial Correspondence to:

Michael Solontoi

Adler Planetarium

1300 S. Lake Shore Drive, Chicago, IL 60605

Email: msolontoi@adlerplanetarium.org 


\section{ABSTRACT}

We present the ensemble properties of 31 comets (27 resolved and 4 unresolved) observed by the Sloan Digital Sky Survey (SDSS). This sample of comets represents about 1 comet per 10 million SDSS photometric objects. Five-band $(u, g, r, i, z)$ photometry is used to determine the comets' colors, sizes, surface brightness profiles, and rates of dust production in terms of the Af $\rho$ formalism. We find that the cumulative luminosity function for the Jupiter Family Comets in our sample is well fit by a power law of the form $N(<H) \propto 10^{(0.49 \pm 0.05) H}$ for $H<18$, with evidence of a much shallower fit $N(<H) \propto 10^{(0.19 \pm 0.03) H}$ for the faint $(14.5<H<18)$ comets. The resolved comets show an extremely narrow distribution of colors $(0.57 \pm 0.05$ in $g-r$ for example), which are statistically indistinguishable from that of the Jupiter Trojans. Further, there is no evidence of correlation between color and physical, dynamical, or observational parameters for the observed comets.

Keywords: COMETS; PHOTOMETRY; COMETS, COMA 


\section{Introduction}

The physical properties of small planetary bodies offer insight into the formation and evolution of the Solar System. However, in situ observational studies of the remote regions of the Solar System are limited to the largest bodies in the Kuiper Belt, and are still not possible in the case of the Oort Cloud. Nevertheless, through delivery of scattered members of their populations, such as comets, these regions may be probed observationally. Comets may be distinguished from other populations of small bodies due to their activity: the production of a gas and dust comae, typically when the comet is at small heliocentric distances, due to the sublimation of volatiles. The comet populations that are thought to directly sample these two remote regions are the Jupiter Family Comets (JFC), from the Kuiper Belt, characterized by generally low-inclination prograde orbits, and Long Period Comets (LPC) thought to originate in the Oort Cloud, with a large range of orbital inclinations, directions, and whose aphelia lie far beyond the orbits of the planets (Jewitt, 2004). Interactions with the giant planets can give rise to comets that exist in-between these two populations, comets with orbits like that of $1 \mathrm{P} /$ Halley. Dynamically, the Tisserand parameter with respect to Jupiter is often employed to distinguish between the various populations of comets based on their orbits (Levison \& Duncan, 1997). Recently a new population of comets has been discovered within the main asteroid belt (Hsieh \& Jewitt, 2006); the orbits of these objects are indistinguishable from those of main belt asteroids, but they have observed activity in the form of comae and dust trails.

Building upon the methodology of detecting active comets in the SDSS described in our previous work (Solontoi et al., 2010), we present here the analysis of properties derived from 35 sets of $u, g, r, i, z$ band photometry of 31 comets observed by the Sloan Digital Sky Survey (York et al., 2000). This includes comets found through the methodology described by Solontoi et al. (2010), along with those recovered through orbital algorithms developed for matching asteroids found in the SDSS to predicted positions of known asteroids (Ivezić et al., 2002; Jurić et al., 2002).

In Section 2 we present a brief overview of the methods employed to find comets in the SDSS. The data and analysis of the observations of both the resolved comets (showing comae), and those that were unresolved (point-source like) are discussed in Section 3. We discuss the color, size and dust properties of the individual comets, as well as the cumulative luminosity and size distributions of the Jupiter Family Comets, in Section 4. 


\section{Observations}

\subsection{Brief Overview of the SDSS}

The data presented here are based on the Seventh SDSS Public Data Release, hereafter DR7 (Abazajian et al., 2009), which ran through July 2008, and contains over 357 million unique photometric objects. Detailed information about this data release including sky coverage, changes from previous data releases and data quality statistics can be found at http://www.sdss.org/DR7, and in Abazajian et al. (2009). Of particular interest to Solar System studies, the survey covers the sky at and near the ecliptic from approximately $\lambda=100^{\circ}$ to $\lambda=225^{\circ}$. The repeat scans of the Southern Galactic hemisphere (Stripe 82; crossing $\lambda=0^{\circ}$ ) also pass through the Ecliptic.

The SDSS is a digital photometric and spectroscopic survey that covered about one quarter of the Celestial Sphere in the North Galactic cap and a smaller $(\sim$ $300 \mathrm{deg}^{2}$ ) but much deeper survey in the Southern Galactic hemisphere and began standard operations in April 2000 (see York et al., 2000; Stoughton et al., 2002; Abazajian et al., 2003, 2004, 2005, 2009; Adelman-McCarthv et al., 2006, 2008). SDSS used a dedicated $2.5 \mathrm{~m}$ telescope (Gunn et al., 2006) to provide homogeneous and deep $(\mathrm{r}<22.5)$ photometry in five band passes (Fukugita et al., 1996; Gunn et al., 1998; Hogg et al., 2001; Smith et al., 2002; Tucker et al., 2006) repeatable to $0.02 \mathrm{mag}$ (root-mean-square scatter, hereafter rms, for sources not limited by photon statistics, Ivezić et al., 2003) and with a zeropoint uncertainty of $\sim 0.02-0.03 \mathrm{mag}$ (Ivezić et al., 2004). The flux densities of detected objects were measured almost simultaneously in five bands $(u, g, r$, $i$, and $z$ ) with effective wavelengths of $3540 \AA, 4760 \AA, 6280 \AA, 7690 \AA$, and $9250 \AA$ (Doi et al., 2010). The large sky coverage of the survey (almost 12,000 $\mathrm{deg}^{2}$ of sky) has resulted in photometric measurements of approximately 357 million objects (Abazaijian et al., 2009). The completeness of SDSS catalogs for point sources is $\sim 99.3 \%$ at the bright end and drops to $95 \%$ at magnitudes of $22.1,22.4,22.1,21.2$, and 20.3 in $u, g, r, i$, and $z$, respectively. Astrometric positions are accurate to better than 0.1 arc-second per coordinate (rms) for sources with $\mathrm{r}<20.5$ (Pier et al., 2003), and the morphological information

from images allows reliable star-galaxy separation to $\mathrm{r} \sim 21.5$ (Lupton et al., 2002; Scranton et al., 2002). A compendium of the technical details about SDSS can be found on the SDSS web site (http://www.sdss.org), which also provides the interface for public data access. 


\subsection{SDSS Photometric Measurements}

When discussing active (resolved) comets, the magnitudes used here are the "model magnitudes" measured by the SDSS. These are designed for galaxy photometry, and are determined by accepting the better of a de Vaucouleurs

and an exponential profile of arbitrary size and orientation (see Stoughton et al., 2002). While the surface brightness profile of a comet differs from that of a galaxy, Solontoi et al. (2010) demonstrate that these models still produce good fits to the cometary data. For the unresolved comets, the magnitudes are based on their point spread function (PSF) magnitude which is measured by fitting the point spread function model to the object. These two magnitudes serve as the basis for discriminating between resolved (galaxy-like) and unresolved (star-like) sources in the SDSS; if the difference in magnitude between the $r$ band PSF magnitude and the $r$ band model magnitude is greater than 0.145 then SDSS classifies the object as resolved, or as a "galaxy" type object (Stoughton et al., 2002).

\subsection{Finding Moving Objects in the SDSS Data}

Although mainly designed for observations of extragalactic sources, the SDSS is significantly contributing to studies of the solar system, notably in the success it has had with asteroid detections, cataloged in the SDSS Moving Object Catalog (hereafter SDSS MOC, Ivezić et al., 2001). This public, valueadded, catalog of SDSS asteroid observations contains, as of its fourth release, 471,000 measurements of moving objects, 220,000 of which have been matched to 104,000 known asteroids from the ASTORB file 1 . The SDSS MOC data is of high quality, and has been widely used in recent studies of asteroids (see Ivezić et al., 2001; Jurić et al., 2002; Binzel et al., 2007; Parker et al., 2008; Assandri \& Gil-Hutton, 2008; Carvano et al., 2010).

The SDSS camera (Gunn et al., 1998) uses a drift-scan-like technique along great circles and detects objects in the order $r, i, u, z, g$, with detection in two successive bands separated in time by 72 seconds, with the different bands registered to the same coordinate system using stationary stars (Pier et al., 2003). Moving objects appear to have their photocenters spacially separated in different filters when color composite images are made. An angular velocity, calculated from the astrometrically-calibrated image centroids in each filter, is calculated for every photometric object.

One of the features that allows the automatic creation of the SDSS MOC is that asteroids appear as point sources. A similar approach to the SDSS

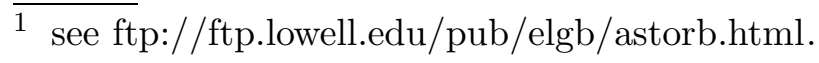


observations of resolved objects, in order to find comets, results in a sample well in excess of a million candidates (Solontoi et al., 2010), vastly larger than even the most optimistic estimate of observable comets. Even if every known comet in the sky were to be imaged, this sample would still be dominated by false positives by a factor of about 1000:1. Therefore, extended objects pose a much more difficult challenge for an automated reduction pipeline, further complicated by the fact that we are searching for moving extended objects, which to the SDSS photometric pipeline are interpreted as "moving galaxies."

\subsection{Finding Comets in the SDSS Data}

In order to successfully acquire comet observations from the SDSS database we employed two methods. The first involved making selection cuts based on SDSS measured photometry and data processing quality flags. The resulting candidate objects were then visually inspected (this method is discussed in

depth by Solontoi et al. 2010). The advantage of this technique is that it is blind to the known comet sample. It does not matter whether the candidates are known comets, thought to be asteroids, or being observed for the first time (i.e. being discovered). The weakness of this approach is that it requires visual identification of each candidate object, and is subject to SDSS pipeline issues (e.g. deblending errors, multiple epochs represented by a single image; see Solontoi et al. 2010 for details). Since this technique was specifically designed for "cometary" (resolved) objects it will not select comets that are inactive, or have sufficiently low activity to appear as point sources at the resolution of the SDSS.

A second technique to select comets from the SDSS employs the method used by Jurić et al. (2002) to identify known asteroids in the SDSS MOC. We utilized the code developed by Jurić et al. (2002) to propagate the orbits of known comets through the SDSS observational cadence. Much as in the case of the SDSS MOC, this code generates all possible RA and dec locations that a known comet could have been observed by the telescope over the course of the survey. This method enabled the selection of both resolved and unresolved comets. Visual inspection of candidates is still required as this list of positions sometimes has large astrometric uncertainties. Comets, unlike asteroids, have significant non-gravitational effects on their motion due to out-gassing during perihelion passage that alter the orbit of a comet over a given apparition, and certainly from passage to passage. For example, Comet Encke returns on average about 2.5 hours sooner than predicted by gravitational computations, a phenomenon that was noticed as far back as the 19th century (Whipple, 1950), and subsequent studies have demonstrated that the majority of periodic comets experience non-gravitational acceleration (Sekanina, 1968). This fact, coupled with many comets not having well calibrated observations over 
several orbits, leads to positional uncertainties in the predicted position. The comet can be too far from the predicted position to be uniquely matched by the algorithm, and in some cases it may not even be present in the SDSS observation field. A second weakness is that the underlying orbital code, based on OrbFit (Milani, 1999) can only be used to find comets with bound (elliptical) orbits, and thus excludes Long Period Comets. The final (and obvious) limitation is that it will only select known comets.

Utilizing these two methods; 35 observations of 31 comets have been identified in the SDSS dataset, including two comets discovered by the survey (C/1999 F2 Dalcanton and C/2000 QJ46 LINEAR). Two additional new comets were observed, but have not been successfully matched to any solar system body with a determined orbit, and thus will not be discussed in this paper; photometry of those two objects is described by Solontoi et al. (2010).

While 35 observations of 31 comets seems small in number compared the typical large number of objects associated with the SDSS, they are in line with what is expected. As discussed in Solontoi et al. (2010), SDSS should be thought of as a single epoch survey in terms of comet results. While different comets are observed on different nights, the sky coverage for a single night is fairly small compared the entire sky, and the cadence of scans is not optimized to follow individual solar system objects. The usable area of the survey for comet work is about $20 \%$ of the whole sky. How many comets should we expect to find in such a survey? The Minor Planet Center 2 lists approximately 200 comets bright enough for robust detection $(r<20)$ on any given night. Taking these 200 potentially observable comets as a typical snapshot of the sky there should be approximately 40 comets in the survey (200 comets on the sky $* 20 \%$ of the sky). Using SDSS DR5 to estimate our completeness (Solontoi et al., 2010) we found it to be about $80 \%$. Taking the sky coverage of SDSS and out completeness into account we expect to find approximately 32 comets, a prediction consistent with 35 observations of 31 comets reported here

Table 1 lists the SDSS $r$ band magnitudes, the difference between the measured PSF and model magnitudes (a point source would be equal to 0) and observing geometry (geocentric and heliocentric distances, and phase angle) for each of the comets discussed in this work.

\section{Analysis}

Here we discuss the methods for determining the photometric colors and surface brightness profiles for the resolved comets. From these measurements,

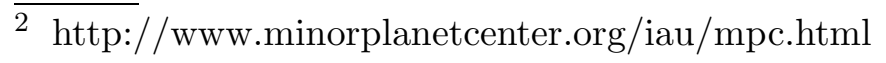


estimates can be made on the upper limit for nuclear radius, and of the dust production via the quantity A $\rho$ (A'Hearn et al., 1984). We then discuss the measurement of photometric colors and radii for the unresolved comets in section 3.2 .

\subsection{Resolved Comets}

In this sample, 31 observations of 27 comets are classified by the SDSS as resolved sources, having a $r$ band PSF-model magnitude value greater than 0.145. For each of these comets the corrected frames (fits files) were obtained from the SDSS Data Archive Server 3 (DAS) for all five filters. Nearby stars and galaxies were masked and radial surface brightness profiles were extracted independently in all five bands for each comet using routines in the IDP3 4 image manipulation package. These profiles consist of azimuthally averaged concentric annuli about the optocenter of the comet in each photometric band. This allows four colors $(u-g, g-r, r-i$, and $i-z)$ to be determined for all comets, even those whose SDSS assigned magnitudes are questionable due to photometric processing errors and non-optimal intensity profile for the flux measurement (see Solontoi et al., 2010, sections 2.3, 3.4 and references therein). In the case of comets with well measured photometry, the total integrated magnitudes determined matched those reported by the SDSS photometric pipeline.

As common with broad band filter studies of comets, we interpret the measured flux and resulting colors as due to the dust coma. Active comets are not free of gas however, and potentially strong emission lines may be present particularly in the wavelength ranges of the $u$ and $g$ bands. To verify these gas emission lines make a negligible contribution to the integrated flux (and therefore color) a sample comet spectrum (Harris et al., 2006) was convolved with the SDSS filter transmission curves (Doi et al., 2010) to produce $u, g$, and $r$-band magnitudes. Compared to a "smoothed" version of the spectrum (one with the emission lines removed) individual magnitudes changed by $3 \%, 0.1 \%$, and $<0.1 \%$ for $u, g$, and $r$-band magnitudes respectively. These changes less than the standard deviation of the measured model magnitudes for the comets in each of these filters.

\subsubsection{Photometric Colors of Resolved Comets}

Extending on the work of Solontoi et al. (2010) we present the SDSS $u-g$, $g-r, r-i$, and $i-z$ colors for the active comets observations in Table 2 .

3 http://das.sdss.org/www/html/

4 http://mips.as.arizona.edu/MIPS/IDP3 
Figures 1 and 2 illustrate the uniformity in the colors of the active comets. Active comets occupy a very narrow distribution in color space, exhibiting the median colors of $u-g: 1.57 \pm 0.21, g-r: 0.57 \pm 0.05, r-i: 0.22 \pm 0.07$, and $i-z: 0.09 \pm 0.07$. To aid in comparison to other Solar System bodies observed in the SDSS, we also calculate the SDSS asteroidal principal component color " $a_{p c}$ " defined as $a_{p c}=0.89(g-r)+0.45(r-i)-0.57$ (Ivezić et al., 2001); the median $a_{p c}$ is $0.04 \pm 0.06$. This value lies between the modes for the $a_{p c}$ color distribution of main-belt asteroids (dominated by $\mathrm{S}$ and $\mathrm{C}$ types), and within uncertainty equal to the median value measured for the Jovian Trojans (Szabó et al., 2007).

When these colors are transformed into BVRI photometric colors (Ivezić et al., 2007) they are shown be consistent with previous color measurements of active comets (see Solontoi et al., 2010, and references within) yeilding mean colors of $\mathrm{B}-\mathrm{V}$ : 0.74, $\mathrm{V}-\mathrm{R}: 0.44$, and $\mathrm{R}-\mathrm{I}: 0.58$. These colors of active comets are slightly bluer (less than $1-\sigma$ ) in $\mathrm{B}-\mathrm{V}$ and $\mathrm{V}-\mathrm{R}$, and redder (less than $2-\sigma$ ) in $\mathrm{R}-\mathrm{I}(-0.09$ in $\mathrm{B}-\mathrm{V},-0.06$ in $\mathrm{V}-\mathrm{R}$, and +0.12 in $\mathrm{R}-\mathrm{I})$ than the colors found for cometary nuclei by Lamy \& Toth (2009).

\subsubsection{Surface Brightness Profiles}

For a simple steady-state coma, the surface brightness should follow a $\rho^{-1}$ relation (Jewitt \& Meech, 1987), with $\rho$ being the projected linear distance from the nucleus. A plot of surface brightness (mag $\operatorname{arcsec}^{-2}$ ) against the $\log$ of angular size, should therefore show a logarithmic gradient $m=-1$. Jewitt \& Meech (1987) observed this relation and showed that when the effects of radiation pressure are taken into account, the slope can become steeper, $(m=-1.5)$. Generally most measurements of the surface brightness profiles of comets tend to fall in the range of $-2<m<-1$ (Jewitt \& Meech, 1987; Lowry et al., 1999, for example). For comets that show profiles steeper than $m=-1.5$ additional factors such as grain fading, may play a role.

The slopes of the observed active SDSS comets' surface brightness profiles were fit using a weighted least-squares method independently in all five SDSS bands; we report these values for all resolved comets in Table 3. Figure 3 shows the surface brightness profiles and fits in all five bands for comet $67 \mathrm{P} /$ ChuryumovGerasimenko, all of which are fit by $m \sim-1$. In addition, the colors are constant over the extent of the comet's profile.

To express our confidence level in the measured surface brightness profiles, we have divided our comets into two "quality groups" based on the value of the difference between their PSF and model magnitudes. Comets that have small PSF-model magnitude values are less well resolved, and the accuracy of the fits to these surface brightness profiles are not as robust as for better resolved 
comets. Figure 4 shows the trend of the mean slope (the mean of the $g, r$ and $i$ slopes) as a function of PSF-model magnitude, and demonstrates a break near PSF-model $=1.5$. At this point, the less resolved comets begin a trend toward steeper slopes that extends all the way down to point-sources (PSFmodel $\sim 0$ ). These point sources (star symbols in Figure 4 ) are stars from the comet fields of similar magnitude and were fit by the same slope fitting routine used for the surface brightness profiles of the comets. Since it is not clear which of the comets are less resolved due to low activity versus those due to the resolution limit of SDSS, we will use a value of PSF-model magnitude difference of 1.5 to define two "quality groups" of surface brightness profile slopes. The well resolved comets (PSF-model $>1.5)$ are placed in QG1 and the less resolved comets $(\mathrm{PSF}-$ model $<1.5)$ in $\mathrm{QG} 2$

For several comets, the $u$ and $z$ bands do not have high enough surface flux density to allow for robust fits to be made, and in those cases no fit is given in Table 3. The $g, r$, and $i$ bands are generally self consistent for a given comet. This trend of similar slopes from the $g, r$, and $i$ band fits is illustrated in Figure 5. The solid lines trace a 1:1 relationship. The fits to the $g, r$, and $i$ band slopes trace each other well, while the fits to the $u$ and $z$ bands show more scatter. The lower quality of fits in the $u$ and $z$ bands is due largely to the surface flux density not being large enough compared to the sky to allow for rigorous fits. This results from a combination of the comets themselves being fainter in the $u$ band, and the lower quantum efficiency of the SDSS camera for the $u$ and $z$ filters (Fukugita et al., 1996; Stoughton et al., 2002).

These five-band surface brightness profiles reveal that not only are the profile slopes consistent across a wide range of wavelengths, as seen in Figure 5 and Table 3, but changes in the profile slope are also consistent from one band to the next. Jewitt \& Meech (1987) predicted a transition to a steeper profile slope at a distance from the nucleus where solar radiation pressure becomes dominant. Several bright comets observed by the SDSS demonstrate this behavior, as illustrated by comet $67 \mathrm{P} / \mathrm{Churyumov-Gerasimenko} \mathrm{in} \mathrm{Figure}$ 3. The slope transition is seen at the same distance (approximately $6 \times 10^{5} \mathrm{~km}$ in the case of $67 \mathrm{P}$ ) for a given comet across all filters where the flux density is significantly above the background.

\subsubsection{Upper Limits to Cometary Radii}

Upper-limit estimates of the radius of the cometary nuclei may be determined by using the PSF fitted magnitude for the resolved comets. We use the measured PSF magnitude from the SDSS photometric pipeline (Stoughton et al., 2002), which involves sync-shifting the image so that it is exactly centered on a pixel, and then fitting a Gaussian model of the PSF to it, with additional corrections applied in order to take into account the full variation of the PSF 
across the field. It would not be useful to take flux from a large aperture for these determinations, as the light from the coma is vastly more luminous than that from the nucleus. While the detected flux in the PSF-magnitude does indeed come from light interacting with the coma, the PSF-magnitude represents the most light that could be theoretically coming from a point source in the region of the nucleus. In a sense this is the same assumption that is made during the calculation of radii from photometry of unresolved comets. An unresolved comet does not show a coma at the level of photometric resolution, and so the PSF photometry of the object is taken to be the light reflecting from the nucleus, even though that light may in fact be from an unresolved coma, rather than the nucleus itself.

Keeping in mind these assumptions, one can take the PSF-magnitude measurement in the $r$ band as a nuclear upper limit, and calculate the absolute magnitude $r_{(1,0)}$ : the $r$ band magnitude the comet would have with a geocentric and heliocentric distance of $1 \mathrm{AU}$ and viewed at a phase angle of zero degrees (this is a physically impossible geometry). So for an observed $r$ band magnitude

$$
r_{(1,0)}=r-5 \log [R \Delta]-\phi(\alpha)
$$

where $R$ and $\Delta$ are the heliocentric and geocentric distances in $\mathrm{AU}, \alpha$ is the observed phase angle, and $\phi(\alpha)$ is the phase function. This absolute magnitude is constructed in the same way using the SDSS $r$ band magnitude as is the standard IAU asteroid $H$ magnitude with the Johnson $V$ band magnitudes (for reference $r_{(1,0)} \sim H-0.2$ ). This absolute magnitude can be written in terms of the comet's diameter, $D$ and albedo, $A$ (Ivezić et al., 2001).

$$
r_{(1,0)}=17.9-2.5 \log \left(\frac{A}{0.1}\right)-5 \log \left(\frac{D}{1 k m}\right)
$$

By assuming a standard albedo of 0.04 and adopting a linear phase function, $\phi(\alpha)=\beta \alpha$, with $\beta=0.035 \mathrm{mag} \mathrm{deg}^{-1}$, these two equations can be used to transform the PSF $r$-band magnitudes into upper limits for the sizes of the comet nuclei. The upper radii limits derived in this manner (Table 4) are comparable to JFC radii in the literature, in particular the list of Jupiter Family Comet size estimates made by Tancredi et al. (2006), who also assume an albedo of 0.04. The trend is that the SDSS upper limits tend to be larger than those published by Tancredi et al. (2006) by about $75 \%$, particularly for the comets for which Tancredi et al. (2006) express high levels of confidence in their estimates.

\subsubsection{Dust Production Rates}

The dust production rate of comets gives insight into the current status, and if measured over time, the evolution of the comet's activity. Dust production 
rates for the observed comets may be characterized from their photometry through the quantity A $f \rho$ defined by A'Hearn et al. (1984). Where A is the dust grain albedo, $f$ the filling factor within the chosen aperture, and $\rho$ the linear radius corresponding to the aperture. Af $\rho$ can be computed directly from observable quantities

$$
A f \rho(\mathrm{cm})=\frac{(2 \Delta R)^{2}}{\rho} \frac{F_{\text {comet }}}{F_{\odot}}
$$

Here $\Delta(\mathrm{cm})$ and $R(\mathrm{AU})$ are the geocentric and heliocentric distances, $\rho(\mathrm{cm})$ is the linear radius of the photometric aperture, and $F_{\text {comet }} / F_{\odot}$ is the ratio of the observed comet flux to that of the Sun. Empirical comparisons indicate a linear relationship between the A $f \rho$ value and the dust production rate, with A $f \rho$ (measured in units of $1000 \mathrm{~cm}$ ) being roughly equal to the modeled dust production rate in metric tons per second (A'Hearn et al., 1995).

For an idealized steady-state coma, the surface brightness profile follows a $\rho^{-1}$ profile. Under that coma model, A $f \rho$ is independent of aperture, but since real comae deviate from $m=-1$ it is necessary to define the radius at which A $f \rho$ is to be measured. Rather than assuming an arbitrary radius we take a cue from galaxy photometry (Petrosian, 1976) and define the radius, $\rho$ at which Af $\rho$ is calculated as the radius at which the local surface brightness value is $10 \%$

of the mean enclosed local surface brightness value, $\frac{S B(\rho)}{\operatorname{mean}[S B(\leq \rho)]}=10 \%$. This criterion results in A $f \rho$ being measured at approximately $10^{3}-10^{5} \mathrm{~km}$ from the center of the comet, and is consistent with radii chosen in the literature for comets over a wide range of observational distances (cf. Lowry et al. 2003 and Mazzotta Epifani et al. 2007).

While under idealized conditions A $f \rho$ is independent of aperture (for a $\rho^{-1}$ profile), and wavelength (for a grey dust approximation), it is dependent on the observational phase angle. By adopting a simple phase function for single dust particles from Divine (1981), the measured Af $\rho$ values may be corrected to $\mathrm{A}(0) f \rho$, the expected value if the comet had been at zero phase angle during the observation. As most reported A $f \rho$ data in literature are not phase corrected, we will generally refer to our uncorrected A $f \rho$ values, unless specifically referencing the phase-adjusted $\mathrm{A}(0) f \rho$. Table 4 lists the $\operatorname{Af} \rho$ and $\mathrm{A}(0) f \rho$ measurements for the active comets.

\subsection{Unresolved Comets}

Four comets in our sample are unresolved by SDSS. Two of these, 19P/Borrelly and $113 \mathrm{P} /$ Spitaler, are dynamically classified as Jupiter Family Comets. 174P/Echeclus is the Centaur 60558 Echeclus that has been observed to have activity (Choi et al., 
2006), and 176P/LINEAR is a main belt comet, previously designated as main belt asteroid 118401 LINEAR (Hsieh \& Jewitt, 2006). All four of these unresolved comets are in the SDSS MOC; 124P and 126P are correctly identified and matched to their asteroid designation, while 19P and 113P, which are in comet but not asteroid databases, are unmatched sources in the SDSS MOC. There are likely to be more unresolved comets to be found in the SDSS MOC, but identifying and retrieving these objects is difficult due to non-gravitational forces causing them to deviate from projected positions on the sky.

The $u-g, g-r, r-i$, and $i-z$ colors are presented in Table 2. Figure 2 shows that the unresolved JFCs, 19P/Borrelly and 113P/Spitaler, fall into the color space spanned by the active comets.

If we assume that these observations of unresolved comets are images of the inactive cometary nucleus, we can make an estimate of the comet's size assuming an albedo of 0.04 . These are included in Table 4, and like the upper limits estimated for the resolved comets, are in satisfactory agreement with previously determined values.

\section{Discussion}

\subsection{Photometric Colors}

In light of the significant differences of observational geometry, physical parameters, and orbital type, all active comets have the same SDSS colors, which span a range of $\sim 6000 \AA$ across the five filters. The photometric $(u-g, g-r$, $r-i$, and $i-z$ ) colors of the active comets (Table 2) reveal that they are remarkably similar to each other, particularly in $g-r, r-i$, and $i-z$, where the colors have a scatter of $\leq 0.07$ magnitude. Furthermore, this uniformity of color does not show systematic variation with observational geometry (phase angle, geocentric or heliocentric distance), measured quantities ( $r$ band magnitude, PSF-model magnitude, slope of the surface brightness profile), derived quantities (nuclear radius, A $f \rho$ ), or orbital properties (orbital inclination, eccentricity and perihelion distance). Figure 6 illustrates the dependence of all four colors on each of these parameters. The colors are consistent with no slope within $1 \sigma$ against all parameters as shown in Figure 7 . The result of the colors of active comets having no correlation with heliocentric distance is consistent with the results of Jewitt \& Meech (1988). Considering that the dust in the coma is thought to be primordial solar system grains freed by the volatilization of ices, this result may indicate that such a color uniformity could be a common property of the primordial dust grains of the outer solar system. 
The colors of the comets discussed here are strikingly similar to that of the Trojans of Jupiter (Szabó et al., 2007) as shown in Figure 2. Trojans also show similar colors to active comets in $5.2-38 \mu \mathrm{m}$ observations Emery et al. (2006), which may indicate a link between the surface chemistry and composition of comets and Trojans.

To further characterize this color similarity we examine the gradient of the normalized reflectivity, $S^{\prime}$, expressed as \% per $1000 \AA$. The colors and distribution of $S^{\prime}$ for the active comets are compared to those of a sample of 363 Trojans, 12 Centaurs, and 23 Trans Neptunian Objects (TNOs) from the SDSS. The Trojan and Centaur samples were selected by their orbital elements (and positive cross-identification) from the SDSS MOC, and the TNOs were found in SDSS Stripe 82 by Becker et al. (2010). Figure 8 shows the range in color-color space occupied by these populations, with the Trojans and active comets being in close agreement. The SDSS colors found in this work agree with those of Szabó et al. (2007), and the $S^{\prime}$ for the active comets resembles

the $S^{\prime}$ distribution of JFC nuclei found by Jewitt (2002). Note that Centaurs are extremely faint objects with correspondingly higher photometric errors.

Histograms of these three populations are seen in Figure 9. The faintness of the Centaurs and TNOs makes the full five-band gradient less reliable. The colors and reflectivity gradients for these populations are summarized in Table 5. By visual inspection, these histograms confirm in $S^{\prime}$ what was seen in their colors: the active comets and Trojans are remarkably similar. To characterize this similarity a Kolmogorov-Smirnov test (KS-test) was performed on the $S^{\prime}$ distributions of the populations in question. Between the Trojans and the Comets, the KS-test probability value was 0.16 with respect to the $S^{\prime}$ distribution across all five bands, and rises to 0.81 for the gri band, suggesting that the comets and the Trojans could have been drawn from the same population. To reiterate, even though Trojans and Comets are two different types of object, with the light we see reflecting off of different sources (solid body vs. dusty coma), their colors are statistically indistinguishable.

\subsection{Nuclear Radii}

Estimates of absolute magnitude and nuclear size were made using equations 1 and 2. Despite the measurements of nuclear radii for the active comets being the result of accepting an upper limit from the SDSS PSF magnitude, the radii presented here agree with the measurements in literature as shown in Table 4.

Even for targeted comet surveys, it is difficult to be certain that all of the flux seen is really light that has interacted with the surface of the comet; in some 
cases there may be low-level activity below the SDSS resolution limit. Certainly there are methods of obtaining highly accurate radius measurements, the best obviously being in situ spacecraft encounters with comets. Only five comets, 1P/Halley (Reinhard, 1986), 19P/Borrelly (Soderblom et al., 2002), 81P/Wild-2 (Brownlee et al., 2004), 9P/Tempel-1 (A'Hearn et al., 2005), and 103P/Hartley-2 (A'Hearn \& DIXI Team, 2011) have to date had observations of the nucleus by remote spacecraft.

Lamy \& Toth (1995) outline how to use the high spatial resolution afforded by the Hubble Space Telescope to disentangle the nucleus signal from the inner coma. Highly accurate results may also be produced if one has access to simultaneous observations of the comet at both visible and infrared wavelengths (Lamy et al., 2002). Most cometary observations, however, must make use of similar methods as employed here. For resolved comets the coma must be algorithmically removed, often either through the modeling of the coma and subtraction, or through the use of small aperture photometry or PSF model fitting. Even unresolved comets must be assumed to be inactive for observation of the radius. Complicating the matter further is the non-uniformity of cometary albedos: while comets are dark overall, the albedo can vary comet-tocomet, and even across the surface of an individual one (Buratti et al., 2004). Being small collisionally-shaped bodies, they can have axial, and even tri-axial shapes. This feature presents an even larger problem as very few comets have had their nuclear light curve accurately measured, (only a few dozen comets have robustly measured nucleus observations) and many of those show dramatic asymmetries (e.g. comet 19P/Borrelly with a primary axis ratio of 2.5:1 (Buratti et al., 2004)).

Although the SDSS resolution is not sufficient for direct measures of the radii they do have the advantage that all observations are made and reduced by a well calibrated and highly characterized process. Thus, the large number of comets observed by the SDSS allows a statistical study of the JFC population, with confidence that the data set is self consistent. To better compare these results with previous cometary studies, we have used the estimated radii upper limits of the comets to calculate (assuming an albedo of 0.04) the more commonly used absolute $H$ magnitude. Figure 10 shows the Cumulative Luminosity Function (CLF - the number of JFCs more luminous than a given $H$ ), and the Cumulative Size Distribution (CSD) for the JFCs. These distributions may be fit with power laws, with the CLF best fit by an exponent of $0.49 \pm 0.05$, and the CSD by $-2.4 \pm 0.2$ for $H<18$. These give the functional form of the distributions as

$$
\begin{gathered}
\text { CLF: } N(<H) \propto 10^{0.49 H} \\
\text { CSD: } N(<r) \propto r^{-2.4}
\end{gathered}
$$


for a given absolute magnitude $H$ and radius $r$. These values are in line with the results of previous studies, which are summarized in Table 6.

We find that these distributions may also be fit with a broken power law, with breaks at $H=14.5$ in the CLF, and at radius $=4 \mathrm{~km}$ in the CSD (Figure 10). For the CLF the gradients are $0.73 \pm 0.08$ for $H<14.5$ and $0.19 \pm 0.03$ for $14.5<H<18$ yielding $-3.1 \pm 0.4$ and $-1.0 \pm 0.1$ for the CSD. This is similar to the CLF measured by Lowry et al. (2003) when considering their entire sample (both resolved and unresolved) of comets $(0.53 \pm 0.04$ and $0.22 \pm 0.02)$. We find that our shallow power law for smaller comets are is agreement, but find that our sample supports a steeper fit for the larger comets. Again these slopes are derived from both radii found for unresolved comets, and upper limits placed on the nuclear radii of resolved comets. While the parameters of this broken power law fit are not strongly constrained they do suggest that that faint comet population could be much shallower than indicated by a single power law fit.

Table 7 compares our results for the Jupiter Family Comets with those of other small body populations. As is the case for many of these populations, the observed CLF power law exponent is similar to 0.5, predicted by Dohnanvi (1969) for a model based on an equilibrium cascade of self-similar collisions. The large number of Main Belt Asteroids observed by the SDSS allows their CLF to be analyzed family-by-family, and analytically fit to a function that accounts for a change in the power law slope (Parker et al., 2008). With the multitude of observations on other small body populations expected from the next generation of sky surveys (e.g. Pan-STARRS and LSST, Kaiser et al., 2002; Ivezić et al., 2008), it is expected that similar detailed analysis could be performed on the less well-sampled populations including Jupiter Family Comets.

\subsection{Af $\rho$}

The A $f \rho$ values obtained for the active comets observed by the SDSS cover a range of three orders of magnitude $0.88 \mathrm{~cm} \leq$ Af $\rho \leq 817.68 \mathrm{~cm}$. Table $8 \mathrm{com}-$ pares the $\operatorname{Af} \rho$ values determined in this paper with those from other studies. They are consistent with other measurements; in particular $67 \mathrm{P} /$ ChuryumovGerasimenko (the ESA Rosetta Mission target) has a well sampled A $f \rho$ over the comet's orbit. Our A $f \rho$ value for this comet is consistent with that found at the same phase angle by Agarwal et al. (2010).

Using Af $\rho$ as proxy for dust production, these values show a trend of the non-JFC comets having significantly higher dust production than do JFC comets. This supports the suggestion that Long Period Comets being recently 
introduced to the inner solar system, are not as surface-volatile depleted as a Jupiter Family Comet that would have presumably made multiple perihelion passages (cf Figure 11). Other factors such as a difference in the size distribution or albedo of the dust between these two populations could also produce a change in $\mathrm{A} f \rho$.

\section{Summary}

We have analyzed data for 31 comets observed in the SDSS, which provides accurately calibrated and measured multi-wavelength photometry in five bands. These comets span a wide range of heliocentric distances, observational, and orbital parameters and all have been observed with the same instrument and processed with the same software. This photometry has been used to make measurements of the colors, sizes, surface brightness profiles and rates of dust production (in terms of the A $f \rho$ formalism). Our main results are as follows.

(1) Despite the variety of cometary parameters, the distributions of photometric colors are extremely narrow $(0.57 \pm 0.05$ in $g-r$ for example), and statistically indistinguishable from those of Jupiter Trojans. The comets exhibit no correlation between color and physical, dynamical, or observational parameters, as seen in Figure 7. Additionally the surface brightness profile for each comet is found to be invariant with wavelength in the optical.

(2) The uniform, red color of the comets indicates that the light from the dust in the coma is complex, no showing any systematic variation with observational geometry, dynamics or physical properties of the comets, and can not be explained through simple scattering (e.g. a simple $1 / \lambda^{n}$ optical depth dependance with $n \sim 4$ ). The visible light observed here is light scattered by the larger particles in the coma, either conglomerates of small grains, or larger, macroscopic rocks. The similarity in reflectance to solid surfaces (both cometary nuclei, and Jupiter Trojans) may indicate that the material responsible for the dark, reddish appearance of these solid objects is also present in the coma and dictates its optical properties.

(3) We find that the cumulative luminosity function for the Jupiter Family Comets can be fit by a power law of the form $N(<H) \propto 10^{(0.49 \pm 0.05) H}$, with evidence of a broken power law with a exponent of $0.73 \pm 0.08$, transitioning to $0.19 \pm 0.03$ at $H \sim 14.5$, suggesting a shallower gradient for the faint population. These results are consistent with distributions of small bodies, both of JFCs, and other Solar System populations.

The analysis presented here is relevant to upcoming large-sky surveys such as the Dark Energy Survey (Flaugher \& Dark Energy Survey Collaboration, 2007), Pan-STARRS (Kaiser et al., 2002) and the Large Synoptic Survey Tele- 
scope (Ivezić et al., 2008, LSST). The results of this work represent a "snapshot" survey of comets, observing a sample of comets at a single point in their orbit. Solontoi et al. (2010) estimate that $10^{3}$ to $10^{4}$ comets will be observed in a survey such as LSST, with a limiting magnitude of $r \sim 24.5$. Further, over the course of 10 years LSST will observe each comet repeatedly. Based on the results of a simulated LSST observational cadence (Ivezić et al., 2008), a JFC such as 70P/Kojima would be observed 300-400 times, spanning its whole heliocentric range. Such observations mean that not only may the analysis presented here be done for a vast number of comets, down to even fainter limiting magnitudes, but the time evolution of each of these parameters may be studied over the comet's orbit.

\section{Acknowledgments}

The authors would like to thank Dr. Yanga Fernández for providing an up to date list of orbital elements for periodic comets, and our two anonymous reviewers for many insightful comments that helped improve the final version.

M.S. would like to acknowledge support from the Brinson Foundation grant in aid of Astrophysics Research to the Adler Planetarium \& Astronomy Museum.

Z.I. acknowledges support by NSF grant AST-0551161 to LSST for design and development activity, and by the Croatian National Science Foundation grant O-1548- 2009.

M.J. wishes to acknowledge the support for this work provided by NASA through Hubble Fellowship grant \#HF-51255.01-A awarded by the Space Telescope Science Institute, which is operated by the Association of Universities for Research in Astronomy, Inc., for NASA, under contract NAS 5-26555.

D.P.S. was supported in part by National Science Foundation grant AST 0607634

IDP3 was developed by the Programming Group for the NICMOS IDT Project (NASA Grant NAG 5-3042, Rodger I. Thompson, P.I.) as a tool for image manipulation and visualization. Many people have contributed to the development of IDP3.

The IDP3 website can be found at http://mips.as.arizona.edu/MIPS/IDP3/.

Funding for the SDSS and SDSS-II has been provided by the Alfred P. Sloan Foundation, the Participating Institutions, the National Science Foundation, the U.S. Department of Energy, the National Aeronautics and Space Administration, the Japanese Monbukagakusho, the Max Planck Society, and 
the Higher Education Funding Council for England. The SDSS Web Site is http://www.sdss.org/.

The SDSS is managed by the Astrophysical Research Consortium for the Participating Institutions. The Participating Institutions are the American Museum of Natural History, Astrophysical Institute Potsdam, University of Basel, University of Cambridge, Case Western Reserve University, University of Chicago, Drexel University, Fermilab, the Institute for Advanced Study, the Japan Participation Group, Johns Hopkins University, the Joint Institute for Nuclear Astrophysics, the Kavli Institute for Particle Astrophysics and Cosmology, the Korean Scientist Group, the Chinese Academy of Sciences (LAMOST), Los Alamos National Laboratory, the Max-Planck-Institute for Astronomy (MPIA), the Max-Planck-Institute for Astrophysics (MPA), New Mexico State University, Ohio State University, University of Pittsburgh, University of Portsmouth, Princeton University, the United States Naval Observatory, and the University of Washington.

\section{References}

Abazajian, K. et al. 2004, AJ , 128, 502

—. 2005, AJ , 129, 1755

-. 2003, AJ , 126, 2081

Abazajian, K. N. et al. 2009, ApJS , 182, 543

Adelman-McCarthy, J. K. et al. 2008, ApJS , 175, 297

-. 2006, ApJS , 162, 38

Agarwal, J., Mueller, M., \& Gruen, E. 2010, ArXiv e-prints

A'Hearn, M. F. et al. 2005, Science, 310, 258

A'Hearn, M. F., \& DIXI Team. 2011, in Bulletin of the American Astronomical Society, Vol. 43, American Astronomical Society Meeting Abstracts \#217, \#306.08-+

A'Hearn, M. F., Millis, R. L., Schleicher, D. G., Osip, D. J., \& Birch, P. V. 1995, Icarus, 118, 223

A'Hearn, M. F., Schleicher, D. G., Millis, R. L., Feldman, P. D., \& Thompson, D. T. 1984 , AJ , 89, 579

Alvarez-Candal, A., \& Licandro, J. 2006, A\&A , 458, 1007

Assandri, M. C., \& Gil-Hutton, R. 2008, A\&A , 488, 339

Becker, A., et al. 2010, in prep

Binzel, R. P. et al. 2007, in Lunar and Planetary Institute Science Conference Abstracts, Vol. 38, Lunar and Planetary Institute Science Conference Abstracts, 1851-+

Bottke, W. F., Jedicke, R., Morbidelli, A., Petit, J., \& Gladman, B. 2000, Science, 288, 2190

Brownlee, D. E. et al. 2004, Science, 304, 1764 
Buratti, B. J., Hicks, M. D., Soderblom, L. A., Britt, D., Oberst, J., \& Hillier, J. K. 2004, Icarus, 167, 16 , special Issue on DS1/Comet Borrelly

Carvano, J. M., Hasselmann, P. H., Lazzaro, D., \& Mothé-Diniz, T. 2010, A\&A , 510, A43+

Choi, Y., Weissman, P. R., \& Polishook, D. 2006, IAU Circ. , 8656, 2

Divine, N. 1981, in ESA Special Publication, Vol. 174, The Comet Halley. Dust and Gas Environment, ed. B. Battrick \& E. Swallow, 47-53

Dohnanyi, J. S. 1969, Journal of Geophysical Research, 74, 2531

Doi, M. et al. 2010, AJ , 139, 1628

Emery, J. P., Cruikshank, D. P., \& van Cleve, J. 2006, Icarus, 182, 496

Fernández, J. A., Tancredi, G., Rickman, H., \& Licandro, J. 1999, A\&A , 352, 327

Flaugher, B., \& Dark Energy Survey Collaboration. 2007, BASS, 209, 22.01

Fukugita, M., Ichikawa, T., Gunn, J. E., Doi, M., Shimasaku, K., \& Schneider, D. P. 1996, AJ , 111, 1748

Gunn, J. E. et al. 1998, AJ , 116, 3040

—. 2006, AJ , 131, 2332

Harris, W. M. et al. 2006, in Bulletin of the American Astronomical Society, Vol. 38, Bulletin of the American Astronomical Society, 502-+

Hogg, D. W., Finkbeiner, D. P., Schlegel, D. J., \& Gunn, J. E. 2001, AJ , 122, 2129

Hsieh, H. H., \& Jewitt, D. 2006, Science, 312, 561

Ivezić, Ž., Juric, M., Lupton, R. H., Tabachnik, S., \& Quinn, T. 2002, in Presented at the Society of Photo-Optical Instrumentation Engineers (SPIE) Conference, Vol. 4836, Society of Photo-Optical Instrumentation Engineers (SPIE) Conference Series, ed. J. A. Tyson \& S. Wolff, 98-103

Ivezić, Ž. et al. 2003, Memorie della Societa Astronomica Italiana, 74, 978

—. 2004, Astronomische Nachrichten, 325, 583

-. 2007, AJ , 134, 973

-. 2001, AJ , 122, 2749

Ivezić, Ž., Tyson, J. A., Allsman, R., Andrew, J., Angel, R., \& for the LSST Collaboration. 2008, ArXiv e-prints, 0805.2366

Jewitt, D. 2006, AJ , 131, 2327

Jewitt, D., \& Meech, K. J. 1988, AJ , 96, 1723

Jewitt, D. C. 2002, AJ , 123, 1039

- 2004, From cradle to grave: the rise and demise of the comets, ed. Festou, M. C., Keller, H. U., \& Weaver, H. A., 659-676

Jewitt, D. C., \& Meech, K. J. 1987, ApJ , 317, 992

Jurić, M. et al. 2002, AJ , 124, 1776

Kaiser, N. et al. 2002, in Presented at the Society of Photo-Optical Instrumentation Engineers (SPIE) Conference, Vol. 4836, Survey and Other Telescope Technologies and Discoveries. Edited by Tyson, J. Anthony; Wolff, Sidney. Proceedings of the SPIE, Volume 4836, pp. 154-164 (2002)., ed. J. A. Tyson \& S. Wolff, 154-164

Lamy, P., \& Toth, I. 2009, Icarus, 201, 674 
Lamy, P. L., \& Toth, I. 1995, A\&A , 293, L43

Lamy, P. L., Toth, I., Fernandez, Y. R., \& Weaver, H. A. 2004, The sizes, shapes, albedos, and colors of cometary nuclei, ed. Festou, M. C., Keller, H. U., \& Weaver, H. A., 223-264

Lamy, P. L., Toth, I., Jorda, L., Groussin, O., A'Hearn, M. F., \& Weaver, H. A. 2002, Icarus, 156, 442

Lamy, P. L., Toth, I., \& Weaver, H. A. 1998, A\&A , 337, 945

Lamy, P. L., Toth, I., Weaver, H. A., A'Hearn, M. F., \& Jorda, L. 2009, A\&A , 508, 1045

Larsen, J. A. et al. 2001, AJ , 121, 562

Levison, H. F., \& Duncan, M. J. 1997, Icarus, 127, 13

Lowry, S. C., \& Fitzsimmons, A. 2001, A\&A , 365, 204

-. 2005, MNRAS , 358, 641

Lowry, S. C., Fitzsimmons, A., Cartwright, I. M., \& Williams, I. P. 1999, A\&A ,349, 649

Lowry, S. C., Fitzsimmons, A., \& Collander-Brown, S. 2003, A\&A , 397, 329

Lupton, R. H., Ivezić, Ž., Gunn, J. E., Knapp, G., Strauss, M. A., \& Yasuda, N. 2002, in Presented at the Society of Photo-Optical Instrumentation Engineers (SPIE) Conference, Vol. 4836, Survey and Other Telescope Technologies and Discoveries. Edited by Tyson, J. Anthony; Wolff, Sidney. Proceedings of the SPIE, Volume 4836, pp. 350-356 (2002)., ed. J. A. Tyson \& S. Wolff, 350-356

Mazzotta Epifani, E., Palumbo, P., Capria, M. T., Cremonese, G., Fulle, M., \& Colangeli, L. 2007, MNRAS , 381, 713

Milani, A. 1999, Icarus, 137, 269

Parker, A., Ivezić, Ž., Jurić, M., Lupton, R., Sekora, M. D., \& Kowalski, A. 2008, Icarus, 198, 138

Petrosian, V. 1976, ApJ , 209, L1

Pier, J. R., Munn, J. A., Hindsley, R. B., Hennessy, G. S., Kent, S. M., Lupton, R. H., \& Ivezić, Ž. 2003, AJ , 125, 1559

Reinhard, R. 1986, Nature , 321, 313

Scranton, R. et al. 2002, ApJ , 579, 48

Sekanina, Z. 1968, Bulletin of the Astronomical Institutes of Czechoslovakia, 19,351

Smith, J. A. et al. 2002, AJ , 123, 2121

Snodgrass, C., Lowry, S. C., \& Fitzsimmons, A. 2006, MNRAS , 373, 1590

-. 2008, MNRAS , 385, 737

Soderblom, L. A. et al. 2002, Science, 296, 1087

Solontoi, M. et al. 2010, Icarus, 205, 605

Stansberry, J., Grundy, W., Brown, M., Cruikshank, D., Spencer, J., Trilling, D., \& Margot, J. 2008, Physical Properties of Kuiper Belt and Centaur Objects: Constraints from the Spitzer Space Telescope, ed. Barucci, M. A., Boehnhardt, H., Cruikshank, D. P., \& Morbidelli, A. , 161-179

Stoughton, C. et al. 2002, AJ , 123, 485

Szabó, G. M., Ivezić, Ž., Jurić, M., \& Lupton, R. 2007, MNRAS , 377, 1393 
Tancredi, G., Fernández, J. A., Rickman, H., \& Licandro, J. 2006, Icarus, 182, 527

Trujillo, C. A., Luu, J. X., Bosh, A. S., \& Elliot, J. L. 2001, AJ , 122, 2740

Tubiana, C., Barrera, L., Drahus, M., \& Boehnhardt, H. 2008, A\&A , 490, 377

Tucker, D. L. et al. 2006, Astronomische Nachrichten, 327, 821

Weissman, P. R., \& Lowry, S. C. 2006, LPI Contributions, 1325, 76

Whipple, F. L. 1950, ApJ , 111, 375

York, D. G. et al. 2000, AJ , 120, 1579 


\begin{tabular}{|c|c|c|c|c|c|}
\hline Comet & $r$ & PSF-model & $\Delta$ & $\mathrm{R}$ & Phase \\
\hline$\dagger 19 \mathrm{P} /$ Borrelly & $21.35 \pm 0.05$ & - & 3.685 & 4.623 & 5.1 \\
\hline 30P Reinmuth & $14.85 \pm 0.01$ & 2.20 & 1.459 & 1.884 & 31.1 \\
\hline 46P/Wirtanen & $18.84 \pm 0.02$ & 1.63 & 1.641 & 2.598 & 7.9 \\
\hline 47P/Ashbrook-Jackson & $20.57 \pm 0.05$ & 0.24 & 3.606 & 4.572 & 2.7 \\
\hline 50P/Arend & $18.13 \pm 0.03$ & 2.35 & 2.574 & 2.809 & 20.8 \\
\hline 62P/Tsuchinshan & $15.04 \pm 0.01$ & 3.91 & 0.953 & 1.904 & 13.1 \\
\hline 64P/Swift-Gehrels & $19.23 \pm 0.04$ & 1.75 & 2.497 & 2.917 & 19 \\
\hline 65P/Gunn (1) & $17.13 \pm 0.01$ & 1.75 & 3.551 & 4.340 & 8.7 \\
\hline 65P/Gunn (2) & $17.15 \pm 0.01$ & 1.90 & 3.559 & 4.338 & 8.9 \\
\hline 67P/Churyumov-Gerasimenko & $14.28 \pm 0.01$ & 3.15 & 1.590 & 1.836 & 32.5 \\
\hline 69P/Taylor & $15.59 \pm 0.01$ & 3.19 & 1.127 & 1.950 & 21.0 \\
\hline 70P/Kojima & $16.68 \pm 0.01$ & 1.97 & 1.629 & 2.594 & 7.6 \\
\hline$\dagger 113 \mathrm{P} /$ Spitaler & $21.05 \pm 0.05$ & - & 2.332 & 3.320 & 2.3 \\
\hline 129P/Shoemaker-Levy & $18.07 \pm 0.02$ & 2.03 & 2.495 & 3.051 & 17.0 \\
\hline 146P/Shoemaker-Linear & $18.78 \pm 0.02$ & 1.27 & 1.301 & 2.023 & 23.9 \\
\hline 158P/Kowal-LINEAR (1) & $18.78 \pm 0.02$ & 1.15 & 3.619 & 4.596 & 3.0 \\
\hline 158P/Kowal-LINEAR (2) & $18.60 \pm 0.02$ & 1.10 & 3.650 & 4.597 & 4.2 \\
\hline 158P/Kowal-LINEAR (3) & $19.36 \pm 0.02$ & 1.01 & 3.988 & 4.798 & 7.3 \\
\hline$\dagger 174 \mathrm{P}$ (60558 Echeclus) & $21.20 \pm 0.08$ & - & 14.350 & 15.167 & 2.27 \\
\hline$\dagger 176 \mathrm{P}$ (118401 LINEAR) & $20.27 \pm 0.03$ & - & 2.374 & 3.256 & 9.2 \\
\hline P/2002 EJ57 (LINEAR) & $18.99 \pm 0.01$ & 0.37 & 1.752 & 2.720 & 5.9 \\
\hline 2003 WY25 & $18.33 \pm 0.01$ & 0.66 & 0.265 & 1.217 & 25.8 \\
\hline C/1999 F2 (Dalcanton) & $15.81 \pm 0.01$ & 2.29 & 4.344 & 4.996 & 9.3 \\
\hline C/2000 K2 (LINEAR) & $16.82 \pm 0.01$ & 1.74 & 3.950 & 4.776 & 7.2 \\
\hline C/2000 QJ46 (LINEAR) (1) & $17.44 \pm 0.01$ & 1.64 & 1.162 & 2.168 & 2.3 \\
\hline C/2000 QJ46 (LINEAR) (2) & $19.39 \pm 0.04$ & 1.47 & 3.318 & 3.657 & 15.3 \\
\hline C/2000 SV74 (LINEAR) & $14.68 \pm 0.00$ & 3.55 & 4.015 & 4.380 & 12.6 \\
\hline C/2000 Y2 (Skiff) & $16.71 \pm 0.01$ & 1.19 & 1.824 & 2.785 & 5.8 \\
\hline C/2001 RX14 (LINEAR) & $12.62 \pm 0.01$ & 2.76 & 1.619 & 2.103 & 26.9 \\
\hline C/2002 O7 (LINEAR) (1) & $19.37 \pm 0.03$ & 1.23 & 5.645 & 5.886 & 9.8 \\
\hline C/2002 O7 (LINEAR) (2) & $15.24 \pm 0.00$ & 2.94 & 2.504 & 3.041 & 17.4 \\
\hline P/1999 V1 (Catalina) & $17.36 \pm 0.01$ & 1.87 & 2.164 & 3.099 & 7.3 \\
\hline P/2002 T5 (LINEAR) & $18.33 \pm 0.02$ & 1.97 & 4.351 & 5.002 & 9.1 \\
\hline P/2004 A1 (LONEOS) & $18.55 \pm 0.01$ & 0.99 & 5.226 & 5.490 & 10.4 \\
\hline P/2006 U5 (Christensen) & $17.63 \pm 0.01$ & 2.04 & 1.538 & 2.358 & 16.6 \\
\hline
\end{tabular}

Table 1

The observed comets. The $r$ band magnitudes reported are SDSS model magnitudes except in the case of unresolved comets (denoted by a $\dagger$ ) where the SDSS PSF magnitudes are reported. $\Delta, \mathrm{R}$, and phase list the Geocentric and Heliocentric distance (AU) and phase angle of the comet at the time of observation. 


\begin{tabular}{|c|c|c|c|c|c|}
\hline Comet & $r$ & $u-g$ & $g-r$ & $r-i$ & $i-z$ \\
\hline \multicolumn{6}{|l|}{ ACTIVE COMETS } \\
\hline 30P Reinmuth & $14.85 \pm 0.01$ & $1.57 \pm 0.02$ & $0.60 \pm 0.01$ & $0.32 \pm 0.01$ & $0.19 \pm 0.01$ \\
\hline 46P/Wirtanen & $18.84 \pm 0.02$ & - & $0.54 \pm 0.09$ & $0.25 \pm 0.05$ & $0.06 \pm 0.05$ \\
\hline 47P/Ashbrook-Jackson & $20.57 \pm 0.05$ & $1.09 \pm 0.33$ & $0.59 \pm 0.07$ & $0.38 \pm 0.07$ & $0.02 \pm 0.17$ \\
\hline 50P/Arend & $18.13 \pm 0.03$ & $1.72 \pm 0.32$ & $0.57 \pm 0.04$ & $0.08 \pm 0.04$ & $0.27 \pm 0.10$ \\
\hline $62 \mathrm{P} /$ Tsuchinshan & $15.04 \pm 0.01$ & $1.27 \pm 0.04$ & $0.45 \pm 0.01$ & $0.18 \pm 0.01$ & $0.07 \pm 0.02$ \\
\hline 64P/Swift-Gehrels & $19.23 \pm 0.04$ & $1.8 \pm 0.45$ & $0.60 \pm 0.06$ & $0.22 \pm 0.06$ & $0.00 \pm 0.21$ \\
\hline 65P/Gunn (1) & $17.13 \pm 0.01$ & $1.68 \pm 0.07$ & $0.58 \pm 0.01$ & $0.21 \pm 0.01$ & $0.08 \pm 0.03$ \\
\hline 65P/Gunn (2) & $17.15 \pm 0.01$ & $1.57 \pm 0.06$ & $0.55 \pm 0.01$ & $0.21 \pm 0.01$ & $0.10 \pm 0.03$ \\
\hline 67P/Churyumov-Gerasimenko & $14.28 \pm 0.01$ & $1.83 \pm 0.03$ & $0.70 \pm 0.01$ & $0.25 \pm 0.01$ & $0.06 \pm 0.01$ \\
\hline 69P/Taylor & $15.59 \pm 0.01$ & $1.51 \pm 0.04$ & $0.60 \pm 0.01$ & $0.25 \pm 0.01$ & $0.06 \pm 0.02$ \\
\hline 70P/Kojima & $16.68 \pm 0.01$ & $1.47 \pm 0.07$ & $0.64 \pm 0.01$ & $0.23 \pm 0.01$ & $0.21 \pm 0.03$ \\
\hline 129P/Shoemaker-Levy & $18.07 \pm 0.02$ & $1.18 \pm 0.12$ & $0.55 \pm 0.03$ & $0.17 \pm 0.03$ & $0.09 \pm 0.07$ \\
\hline 146P/Shoemaker-Linear & $18.78 \pm 0.02$ & $1.46 \pm 0.15$ & $0.64 \pm 0.03$ & $0.25 \pm 0.03$ & $0.12 \pm 0.06$ \\
\hline 158P/Kowal-LINEAR (1) & $18.78 \pm 0.02$ & $1.59 \pm 0.19$ & $0.56 \pm 0.03$ & $0.22 \pm 0.03$ & $0.01 \pm 0.09$ \\
\hline 158P/Kowal-LINEAR (2) & $18.60 \pm 0.02$ & $1.48 \pm 0.14$ & $0.59 \pm 0.03$ & $0.19 \pm 0.02$ & $0.20 \pm 0.05$ \\
\hline 158P/Kowal-LINEAR (3) & $19.36 \pm 0.02$ & $2.09 \pm 0.39$ & $0.58 \pm 0.03$ & $0.20 \pm 0.04$ & $0.19 \pm 0.08$ \\
\hline P/2002 EJ57 (LINEAR) & $18.99 \pm 0.01$ & $1.57 \pm 0.11$ & $0.54 \pm 0.02$ & $0.20 \pm 0.02$ & $0.12 \pm 0.05$ \\
\hline 2003 WY25 & $18.33 \pm 0.01$ & $1.47 \pm 0.08$ & $0.64 \pm 0.01$ & $0.27 \pm 0.01$ & $0.12 \pm 0.04$ \\
\hline C/1999 F2 (Dalcanton) & $15.81 \pm 0.01$ & $1.69 \pm 0.05$ & $0.52 \pm 0.07$ & $0.25 \pm 0.05$ & $0.07 \pm 0.05$ \\
\hline C/2000 K2 (LINEAR) & $16.82 \pm 0.01$ & $1.55 \pm 0.06$ & $0.55 \pm 0.01$ & $0.22 \pm 0.01$ & $0.10 \pm 0.02$ \\
\hline C/2000 QJ46 (LINEAR) (1) & $17.44 \pm 0.01$ & $1.55 \pm 0.11$ & $0.57 \pm 0.02$ & $0.23 \pm 0.01$ & $0.12 \pm 0.04$ \\
\hline C/2000 QJ46 (LINEAR) (2) & $19.39 \pm 0.04$ & $1.04 \pm 0.27$ & $0.56 \pm 0.06$ & $0.41 \pm 0.06$ & $0.09 \pm 0.13$ \\
\hline C/2000 SV74 (LINEAR) & $14.68 \pm 0.00$ & $1.66 \pm 0.02$ & $0.52 \pm 0.01$ & $0.21 \pm 0.01$ & $0.04 \pm 0.01$ \\
\hline C/2000 Y2 (Skiff) & $16.71 \pm 0.01$ & $1.58 \pm 0.04$ & $0.55 \pm 0.01$ & $0.18 \pm 0.01$ & $0.09 \pm 0.01$ \\
\hline C/2001 RX14 (LINEAR) & $12.62 \pm 0.01$ & $1.61 \pm 0.03$ & $0.57 \pm 0.02$ & $0.23 \pm 0.02$ & $0.06 \pm 0.01$ \\
\hline C/2002 O7 (LINEAR) (1) & $19.37 \pm 0.03$ & $1.35 \pm 0.21$ & $0.58 \pm 0.04$ & $0.14 \pm 0.04$ & $-0.12 \pm 0.25$ \\
\hline C/2002 O7 (LINEAR) (2) & $15.24 \pm 0.01$ & $1.56 \pm 0.07$ & $0.56 \pm 0.02$ & $0.22 \pm 0.02$ & $0.07 \pm 0.03$ \\
\hline P/1999 V1 (Catalina) & $17.36 \pm 0.01$ & $1.73 \pm 0.09$ & $0.58 \pm 0.01$ & $0.23 \pm 0.01$ & $0.09 \pm 0.03$ \\
\hline P/2002 T5 (LINEAR) & $18.33 \pm 0.02$ & $1.52 \pm 0.21$ & $0.66 \pm 0.03$ & $0.23 \pm 0.03$ & $0.09 \pm 0.10$ \\
\hline P/2004 A1 (LONEOS) & $18.55 \pm 0.01$ & $1.67 \pm 0.20$ & $0.61 \pm 0.02$ & $0.31 \pm 0.02$ & $0.09 \pm 0.05$ \\
\hline P/2006 U5 (Christensen) & $17.63 \pm 0.01$ & $1.46 \pm 0.09$ & $0.51 \pm 0.01$ & $0.08 \pm 0.01$ & $0.22 \pm 0.04$ \\
\hline Median Color & & $1.57 \pm 0.21$ & $0.57 \pm 0.05$ & $0.22 \pm 0.07$ & $0.09 \pm 0.07$ \\
\hline \multicolumn{6}{|l|}{ UNRESOLVED COMETS } \\
\hline 19P/Borrelly & $21.35 \pm 0.05$ & $1.11 \pm 0.50$ & $0.77 \pm 0.09$ & $0.12 \pm 0.09$ & $0.27 \pm 0.25$ \\
\hline 113P/Spitaler & $21.05 \pm 0.05$ & $1.06 \pm 0.44$ & $0.60 \pm 0.08$ & $0.33 \pm 0.07$ & $0.00 \pm 0.61$ \\
\hline 174P (60558 Echeclus) & $21.20 \pm 0.08$ & $1.55 \pm 1.02$ & $0.73 \pm 0.13$ & $0.18 \pm 0.08$ & $-0.71 \pm 0.53$ \\
\hline 176P (118401 LINEAR) & $20.27 \pm 0.03$ & $1.67 \pm 0.40$ & $0.54 \pm 0.04$ & $0.06 \pm 0.05$ & $0.04 \pm 0.10$ \\
\hline
\end{tabular}

Table 2

The SDSS colors of the comets. The listed $r$ band magnitudes are SDSS model magnitudes for active comets, and SDSS PSF magnitudes for the unresolved comets. 


\begin{tabular}{|c|c|c|c|c|c|c|c|}
\hline \multirow[t]{2}{*}{ Comet } & \multirow[t]{2}{*}{ Psf-model } & \multicolumn{6}{|c|}{ Surface Brightness Profile Slope } \\
\hline & & & $u$ & $g$ & $r$ & $i$ & $z$ \\
\hline 30P Reinmuth & 2.20 & -1.57 & $7 \pm 0.16$ & $-1.57 \pm 0.01$ & $-1.58 \pm 0.01$ & $-1.54 \pm 0.01$ & $-1.55 \pm 0.01$ \\
\hline 46P/Wirtanen & 1.63 & & - & $-1.18 \pm 0.06$ & $-1.22 \pm 0.06$ & $-1.34 \pm 0.06$ & $-1.54 \pm 0.40$ \\
\hline 47P/Ashbrook-Jackson & 0.24 & & - & $-1.87 \pm 0.26$ & $-2.85 \pm 0.28$ & $-1.74 \pm 0.06$ & $-2.43 \pm 0.31$ \\
\hline 50P/Arend & 2.35 & & - & $-1.31 \pm 0.07$ & $-1.38 \pm 0.11$ & $-1.50 \pm 0.15$ & $-1.32 \pm 0.29$ \\
\hline $62 \mathrm{P} /$ Tsuchinshan & 3.91 & -0.91 & $1 \pm 0.07$ & $-0.86 \pm 0.01$ & $-0.97 \pm 0.01$ & $-0.98 \pm 0.01$ & $-0.90 \pm 0.03$ \\
\hline $64 \mathrm{P} /$ Swift-Gehrels & 1.75 & & - & $-1.29 \pm 0.18$ & $-1.22 \pm 0.11$ & $-1.28 \pm 0.16$ & - \\
\hline 65P/Gunn (1) & 1.75 & -1.58 & $8 \pm 0.10$ & $-1.88 \pm 0.05$ & $-1.94 \pm 0.04$ & $-1.94 \pm 0.04$ & $-1.66 \pm 0.17$ \\
\hline 65P/Gunn (2) & 1.90 & -2.27 & $7 \pm 0.56$ & $-1.87 \pm 0.03$ & $-1.91 \pm 0.04$ & $-1.82 \pm 0.04$ & $-1.73 \pm 0.07$ \\
\hline 67P/Churyumov-Gerasimenko & 3.15 & -0.99 & $9 \pm 0.04$ & $-1.05 \pm 0.01$ & $-1.04 \pm 0.01$ & $-1.02 \pm 0.01$ & $-1.05 \pm 0.02$ \\
\hline 69P/Taylor & 3.19 & -1.38 & $8 \pm 0.24$ & $-1.24 \pm 0.01$ & $-1.35 \pm 0.01$ & $-1.33 \pm 0.01$ & $-1.42 \pm 0.04$ \\
\hline 70P/Kojima & 1.97 & -0.97 & $7 \pm 0.41$ & $-1.32 \pm 0.03$ & $-1.41 \pm 0.02$ & $-1.43 \pm 0.02$ & $-1.39 \pm 0.06$ \\
\hline 129P/Shoemaker-Levy & 2.03 & & - & $-1.85 \pm 0.10$ & $-1.67 \pm 0.04$ & $-1.53 \pm 0.07$ & $-1.13 \pm 0.20$ \\
\hline 146P/Shoemaker-LINEAR & 1.27 & & - & $-1.62 \pm 0.12$ & $-1.74 \pm 0.11$ & $-1.74 \pm 0.10$ & $-1.75 \pm 0.42$ \\
\hline 158P/Kowal-LINEAR (1) & 1.15 & & - & $-1.99 \pm 0.11$ & $-2.20 \pm 0.10$ & $-2.03 \pm 0.15$ & $-2.10 \pm 0.19$ \\
\hline 158P/Kowal-LINEAR (2) & 1.10 & -2.28 & $8 \pm 0.59$ & $-2.30 \pm 0.11$ & $-2.30 \pm 0.08$ & $-2.08 \pm 0.10$ & $-2.79 \pm 0.49$ \\
\hline 158P/Kowal-LINEAR (3) & 1.01 & & - & $-2.12 \pm 0.17$ & $-1.85 \pm 0.07$ & $-1.83 \pm 0.11$ & $-1.77 \pm 0.33$ \\
\hline P/2002 EJ57 (LINEAR) & 0.37 & -1.87 & $7 \pm 0.36$ & $-2.84 \pm 0.18$ & $-2.96 \pm 0.14$ & $-3.01 \pm 0.12$ & $-3.14 \pm 0.27$ \\
\hline 2003 WY25 & 0.66 & -1.24 & $4 \pm 0.39$ & $-1.88 \pm 0.07$ & $-1.76 \pm 0.06$ & $-2.04 \pm 0.09$ & $-1.90 \pm 0.17$ \\
\hline C/1999 F2 (Dalcanton) & 2.29 & -1.12 & $2 \pm 0.10$ & $-1.08 \pm 0.01$ & $-1.07 \pm 0.01$ & $-1.03 \pm 0.01$ & $-1.07 \pm 0.03$ \\
\hline C/2000 K2 (LINEAR) & 1.74 & -2.03 & $3 \pm 0.41$ & $-1.94 \pm 0.03$ & $-1.92 \pm 0.03$ & $-1.89 \pm 0.02$ & $-1.71 \pm 0.05$ \\
\hline C/2000 QJ46 (LINEAR) (1) & 1.64 & -2.05 & $5 \pm 0.35$ & $-1.69 \pm 0.04$ & $-1.73 \pm 0.02$ & $-1.89 \pm 0.04$ & $-1.95 \pm 0.13$ \\
\hline C/2000 QJ46 (LINEAR) (2) & 1.47 & & - & $-1.96 \pm 0.32$ & $-1.49 \pm 0.21$ & $-1.96 \pm 0.44$ & $-1.96 \pm 0.60$ \\
\hline C/2000 SV74 (LINEAR) & 3.55 & -1.19 & $9 \pm 0.17$ & $-1.24 \pm 0.01$ & $-1.23 \pm 0.01$ & $-1.22 \pm 0.01$ & $-1.20 \pm 0.02$ \\
\hline C/2000 Y2 (Skiff) & 1.19 & -2.08 & $8 \pm 0.22$ & $-2.11 \pm 0.02$ & $-2.17 \pm 0.03$ & $-2.08 \pm 0.04$ & $-1.94 \pm 0.06$ \\
\hline C/2001 RX14 (LINEAR) & 2.76 & -1.31 & $1 \pm 0.01$ & $-1.39 \pm 0.00$ & $-1.40 \pm 0.01$ & $-1.36 \pm 0.00$ & $-1.35 \pm 0.01$ \\
\hline C/2002 O7 (LINEAR) (1) & 1.23 & & - & $-2.41 \pm 0.10$ & $-2.49 \pm 0.28$ & $-2.01 \pm 0.22$ & $-1.06 \pm 0.34$ \\
\hline C/2002 O7 (LINEAR) (2) & 2.94 & -1.37 & $7 \pm 0.05$ & $-1.49 \pm 0.04$ & $-1.47 \pm 0.04$ & $-1.40 \pm 0.05$ & $-1.37 \pm 0.05$ \\
\hline P/1999 V1 (Catalina) & 1.87 & -1.03 & $3 \pm 0.06$ & $-1.64 \pm 0.03$ & $-1.60 \pm 0.03$ & $-1.65 \pm 0.03$ & $-1.55 \pm 0.10$ \\
\hline P/2002 T5 (LINEAR) & 1.97 & & - & $-1.68 \pm 0.11$ & $-1.70 \pm 0.08$ & $-1.51 \pm 0.05$ & $-1.34 \pm 0.53$ \\
\hline P/2004 A1 (LONEOS) & 0.99 & & - & $-2.41 \pm 0.09$ & $-2.51 \pm 0.13$ & $-2.44 \pm 0.14$ & $-1.98 \pm 0.29$ \\
\hline P/2006 U5 (Christensen) & 2.04 & -1.87 & $7 \pm 0.48$ & $-1.57 \pm 0.05$ & $-1.59 \pm 0.03$ & $-1.70 \pm 0.03$ & $-1.46 \pm 0.06$ \\
\hline
\end{tabular}

\section{Table 3}

Linear fits of the Surface Brightness Profile slopes of the active comets in all five SDSS bands, described in Section 3.1.2. PSF-model is the difference between the SDSS measured PSF and model magnitudes 


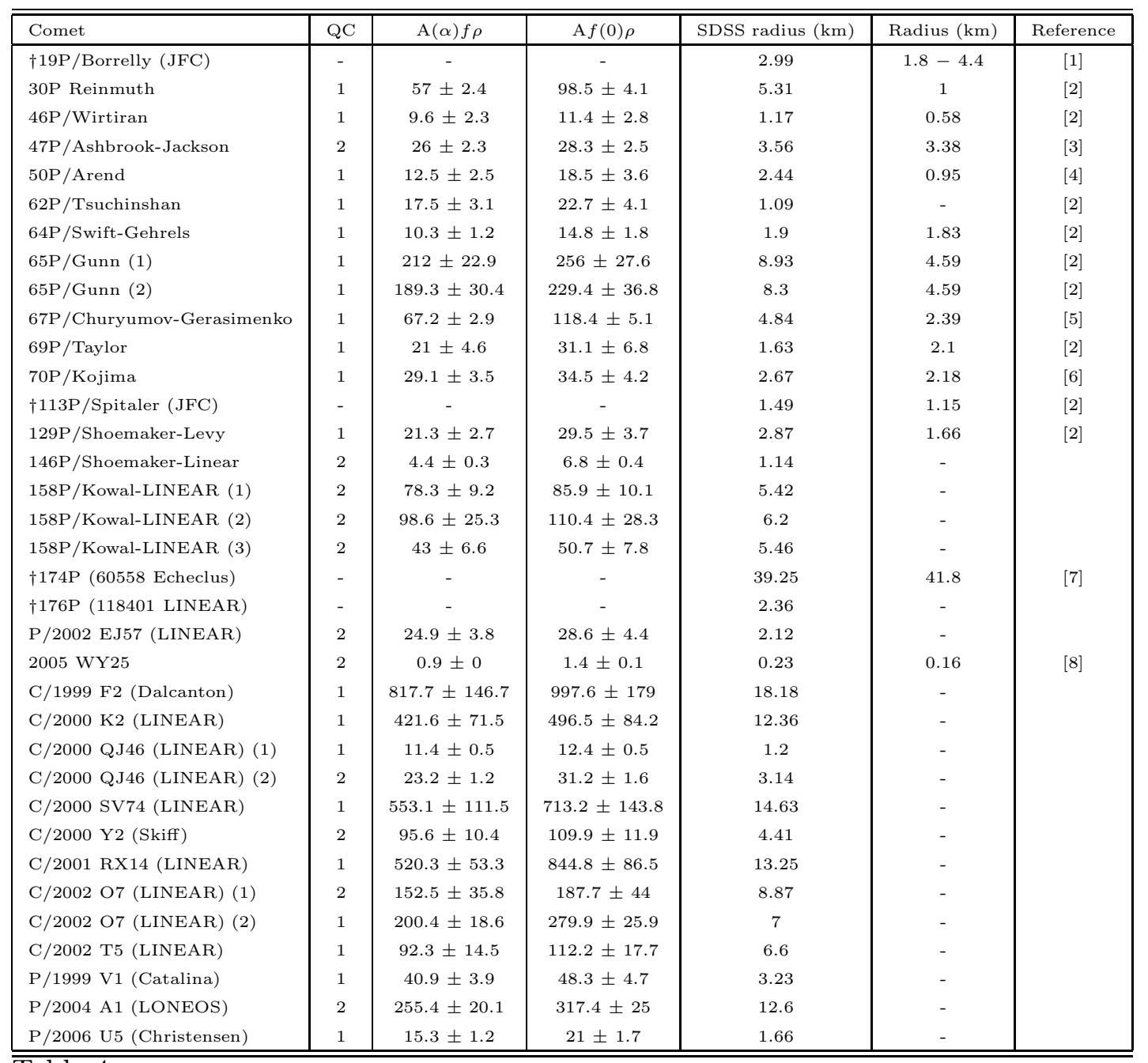

\section{Table 4}

Derived quantities. Af $\rho$ values are those derived from SDSS observations; A(0) f $\rho$ entries are corrected for a phase angle of $\alpha=0$ (see Divine, 1981; Agarwal et al., 2010). The SDSS radii are all upper limits except for the unresolved comets marked with a $\dagger$. Literature radii values from [1] Lamv et al. (1998), [2] Tancredi et al. (2006), [3] Snodgrass et al. (2006), [4] Lamv et al. (2009), [5] Tubiana et al. (2008), [6] Snodgrass et al. (2008), [7] Stansberry et al. (2008), and [8] Jewitt (2006)

\begin{tabular}{|l|c|c|c|c|c|c|}
\hline \hline Population & $u-g$ & $g-r$ & $r-i$ & $i-z$ & $S^{\prime}(u, g, r, i, z)$ & $S^{\prime}(g, r, i)$ \\
\hline Comets & $1.57 \pm 0.21$ & $0.57 \pm 0.05$ & $0.22 \pm 0.07$ & $0.09 \pm 0.07$ & $8.4 \pm 3.6$ & $8.3 \pm 3.5$ \\
Trojans & $1.47 \pm 0.17$ & $0.58 \pm 0.07$ & $0.23 \pm 0.5$ & $0.13 \pm 0.08$ & $8.2 \pm 2.9$ & $8.8 \pm 2.9$ \\
Centaurs & $1.41 \pm 0.46$ & $0.62 \pm 0.16$ & $0.26 \pm 0.17$ & $0.21 \pm 0.16$ & $8.9 \pm 7.7$ & $10.3 \pm 11.1$ \\
TNO & $1.03 \pm 1.21$ & $0.74 \pm 0.40$ & $0.35 \pm 0.26$ & $0.18 \pm 0.17$ & $16.4 \pm 9.2$ & $17.8 \pm 11.8$ \\
\hline \hline
\end{tabular}

\section{Table 5}

Colors and spectral gradient of populations as measured by the SDSS. The colors of the comets, centaurs, and Trojans, and TNOs here are those of the bodies used to calculate the $S^{\prime}$, as discussed in section 4.1. The TNO colors are from Becker et al. (2010). 
Table 6

\begin{tabular}{|l|c|c|}
\hline \hline Reference & JFC CLF & JFC CSD \\
\hline This Work & $0.49 \pm 0.05$ & $-2.4 \pm 0.2$ \\
Tancredi et al. (2006) & $0.53 \pm 0.05$ & $-2.7 \pm 0.3$ \\
Weissman \& Lowrv (2006) & $0.35 \pm 0.01$ & $-1.73 \pm 0.06$ \\
Lamv et al. (2004) & $0.38 \pm 0.06$ & $-1.9 \pm 0.3$ \\
Lowry et al. (2003) & $0.32 \pm 0.02$ & $-1.6 \pm 0.1$ \\
Fernández et al. (1999) & $0.53 \pm 0.05$ & $-2.65 \pm 0.25$ \\
\hline \hline
\end{tabular}

Estimates of the cumulative luminosity function and cumulative size distribution power law slopes for the Jupiter Family Comets.

\begin{tabular}{|l|c|c|c|l|}
\hline \hline Population & Broken Min & Single Power Law & Broken Max & Source \\
\hline$\dagger$ Jupiter Family Comets & $0.19 \pm 0.03$ & $0.49 \pm 0.05$ & $0.73 \pm 0.08$ & This Work \\
Near Earth Asteroids & & $0.35 \pm 0.02$ & & Bottke et al. (2000) \\
Asteroids (cometary orbits) & & $0.51 \pm 0.02$ & & Alvarez-Candal \& Licandro (2006) \\
$\dagger$ Main Belt Asteroids (broken) & $0.10-0.59$ & $0.35-0.97$ & $0.37-1.04$ & Parker et al. (2008) \\
Hildas & & $0.42 \pm 0.02$ & & Alvarez-Candal \& Licandro (2006) \\
$\dagger$ Trojans & & $0.44 \pm 0.05$ & & Szabó et al. (2007) \\
Centaurs & & $0.54 \pm 0.07$ & & Larsen et al. (2001) \\
Kuiper Belt Objects & & $0.66 \pm 0.06$ & & Truiillo et al. (2001) \\
\hline
\end{tabular}

\section{Table 7}

Estimates of the cumulative luminosity function power laws for small-body populations. The CLF of Main Belt Asteroid are family populations that are fit both by broken and unbroken power laws, with the values cited here being the ranges determined for these families in Parker et al. (2008). Those populations marked by a $\dagger$ are derived from SDSS data.

\begin{tabular}{|l|c|c|c|c|c|}
\hline \hline Comet & $\mathrm{R}(\mathrm{AU})$ & $\mathrm{A} f \rho(\mathrm{cm})$ & Previous R $(\mathrm{AU})$ & Previous A $f \rho(\mathrm{cm})$ & Source \\
\hline $46 \mathrm{P} /$ Wirtanen & 2.598 & $9.59 \pm 2.35$ & 1.120 & 112.2 & 1 \\
$47 \mathrm{P} /$ Ashbrook-Jackson & 4.572 & $25.97 \pm 2.28$ & 4.030 & $28.95 \pm 4.1$ & 2 \\
$65 \mathrm{P} /$ Gunn & 4.340 & $211.97 \pm 22.86$ & 4.430 & $133.4 \pm 4.7$ & 3 \\
& 4.338 & $189.28 \pm 30.37$ & 2.640 & 23.4 & 1 \\
$\dagger 67 \mathrm{P} /$ Churyumov-Gerasimenko & 1.836 & $67.21 \pm 2.9$ & 1.380 & 208.9 & 1 \\
$69 \mathrm{P} /$ Taylor & 1.950 & $21 \pm 4.59$ & 4.030 & $18.39 \pm 1.7$ & 2 \\
129P/Shoemaker-Levy & 3.051 & $21.26 \pm 2.68$ & 4.558 & $4.5 \pm 0.6$ & 4 \\
\hline \hline
\end{tabular}

Table 8

A comparison of our A $f \rho$ values with previous listed values. References: [1] A'Hearn et al. (1995), [2] Lowry et al. (2003), [3] Lowry \& Fitzsimmons (2001), [4] Lowry \& Fitzsimmons (2005). ( $\nmid$ A detailed look at Af $\rho$ for $67 \mathrm{P} /$ ChuryumovGerasimenko can be found in Agarwal et al. (2010). 


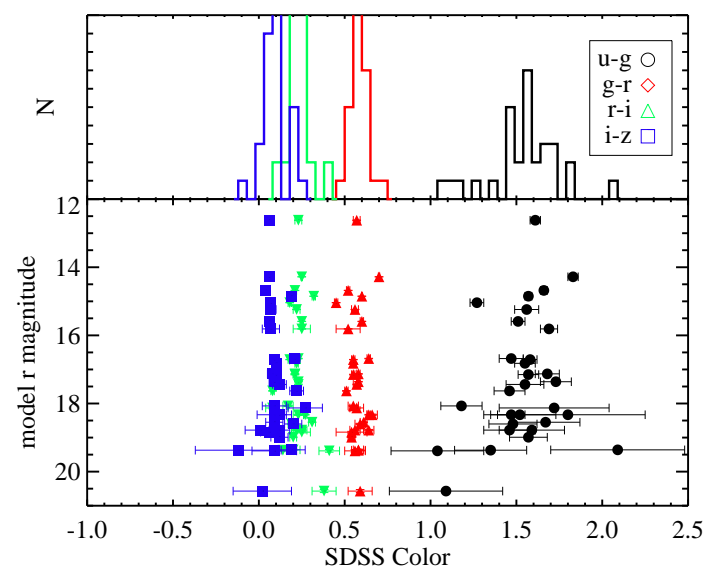

Fig. 1. Color-magnitude distribution of the resolved comets with 2- $\sigma$ error bars. The plotted symbols are: $\bigcirc u-g$ (black), $\triangle g-r$ (red), $\nabla r-i$ (green), and $\square i-z$ (blue). Plotted above is the histogram of the color distribution.
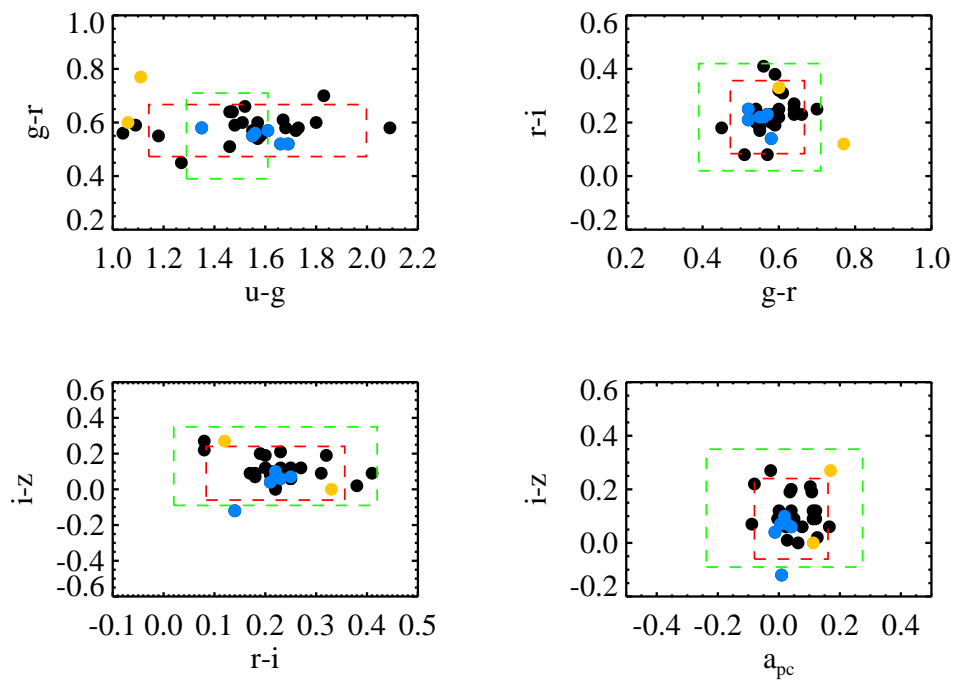

Fig. 2. Color-color plots, showing the distribution of the SDSS comets. The resolved Jupiter Family Comets (JFC) are in black, while non-JFC resolved comets are in blue. The boxes represent the range of 2 standard deviations for the resolved comet distribution (red), and Jupiter Trojans (green) from Szabó et al. (2007). $a_{p c}$ is the principle component color in the MOC defined as $a_{p c}=0.89(g-r)+0.45(r-i)-0.57$ (see Ivezić et al. 2001). The two unresolved JFCs are in orange. 

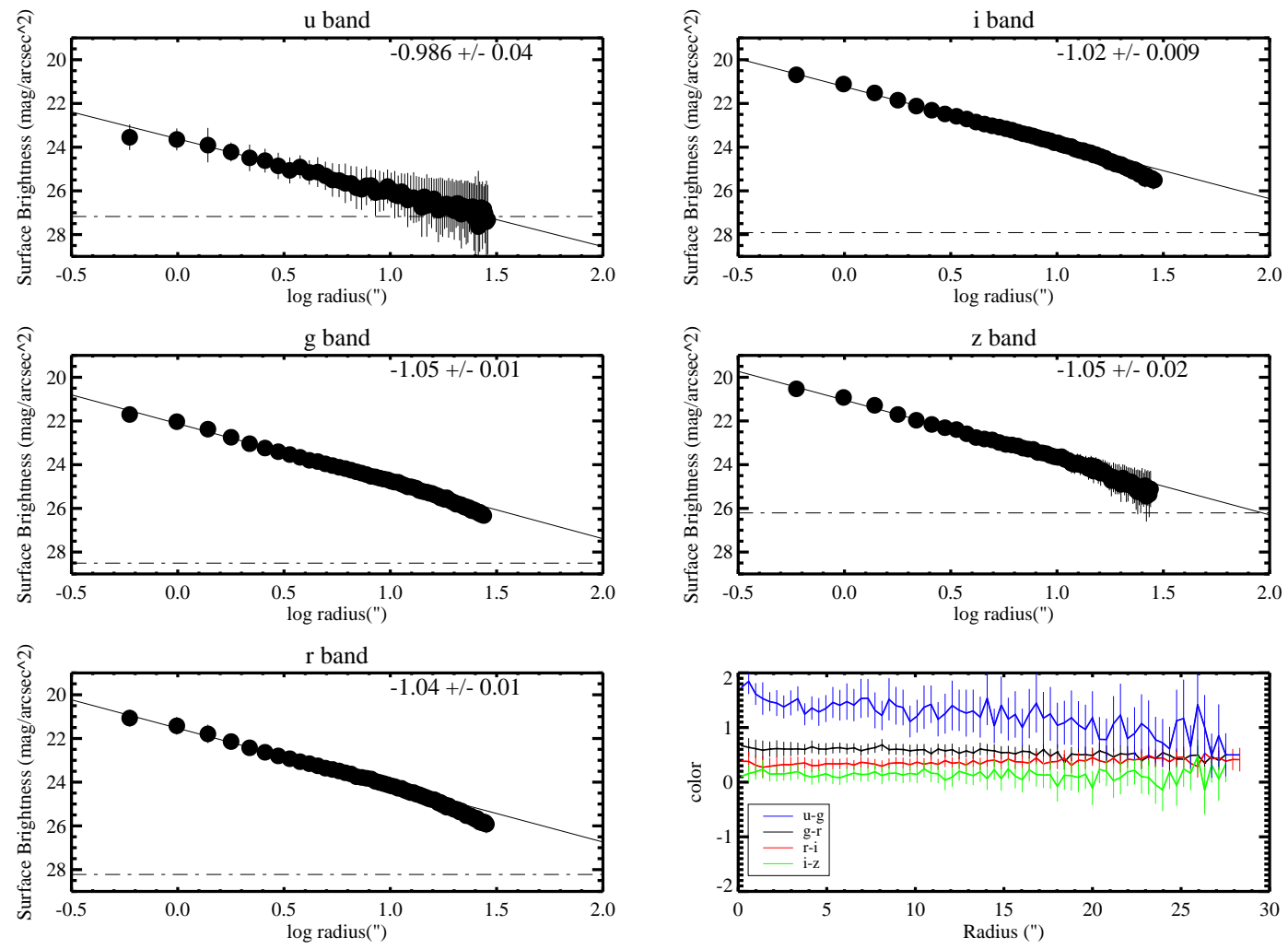

Fig. 3. Surface brightness profile of comet $67 \mathrm{P} /$ Churyumov-Gerasimenko in $u, g, r, i, z$, along with the weighted least squares fit for the slope (solid black line, with value in each panel's upper right). One count above sky would give the surface brightness shown by the horizontal dashed lines. The bottom right panel shows the local $u-g, g-r, r-i$ and $i-z$ colors with respect to radius. In all cases error bars are $1-\sigma$. The color remains consistent with respect to radius until the local surface brightness becomes close to sky values (most prominently seen with $u-g$ in blue) 


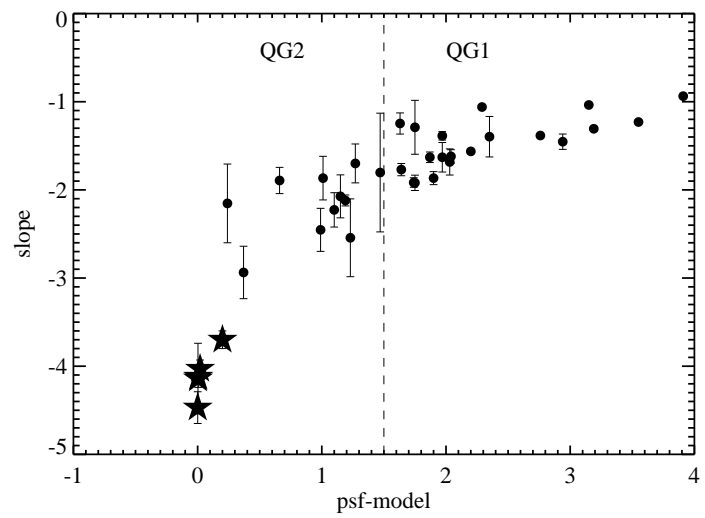

Fig. 4. The average slope of the $u, g$, and $r$ band surface brightness profiles plotted against the PSF-model magnitudes of the active comets. We divide the sample into two "quality groups" (QG1 and QG2) at PSF-model $=1.5$. For the more resolved QG1 comets the trend is fairly flat, and is characterized by generally small errors in the slope fits. The less resolved QG2 comets trend toward steeper slopes, a trend that terminates with slopes fit to point-sources, as shown by the four stars in the plot. The four objects near PSF-model $=0$ (star symbols) are field stars whose surface brightness profiles were extracted and fit in the same manner as the comets.
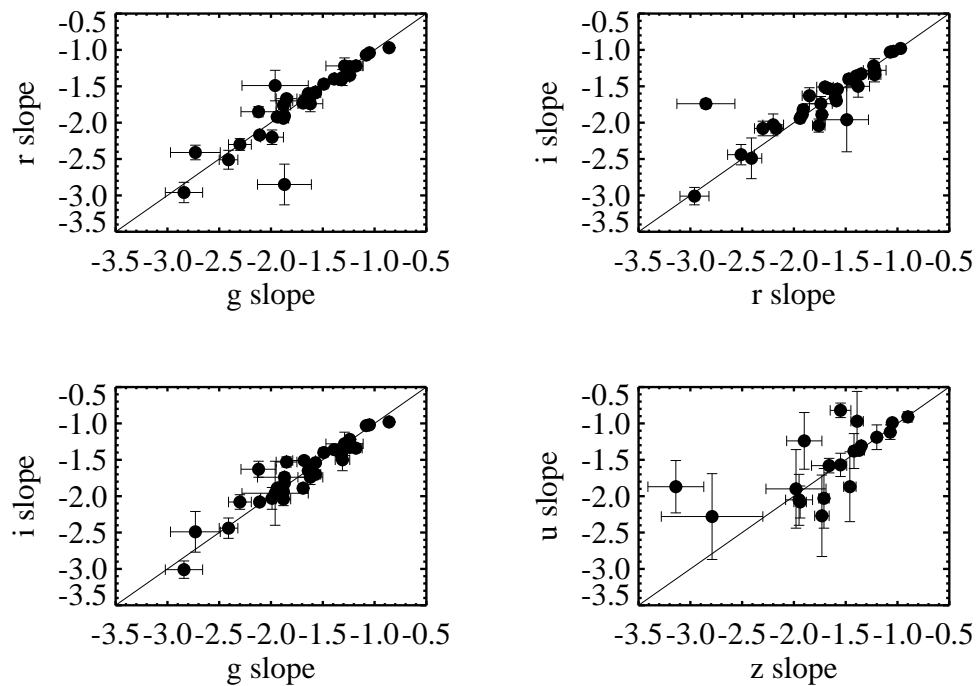

Fig. 5. Comparison between the best fit slopes of the surface brightness profiles for the SDSS filters. The solid lines are the 1:1 relations. Trends between the $g, r$ and $i$ band slopes are nearly 1:1, while there is more scatter in the $u$ and $z$ band trends. The plotted points carry $1 \sigma$ error bars. 

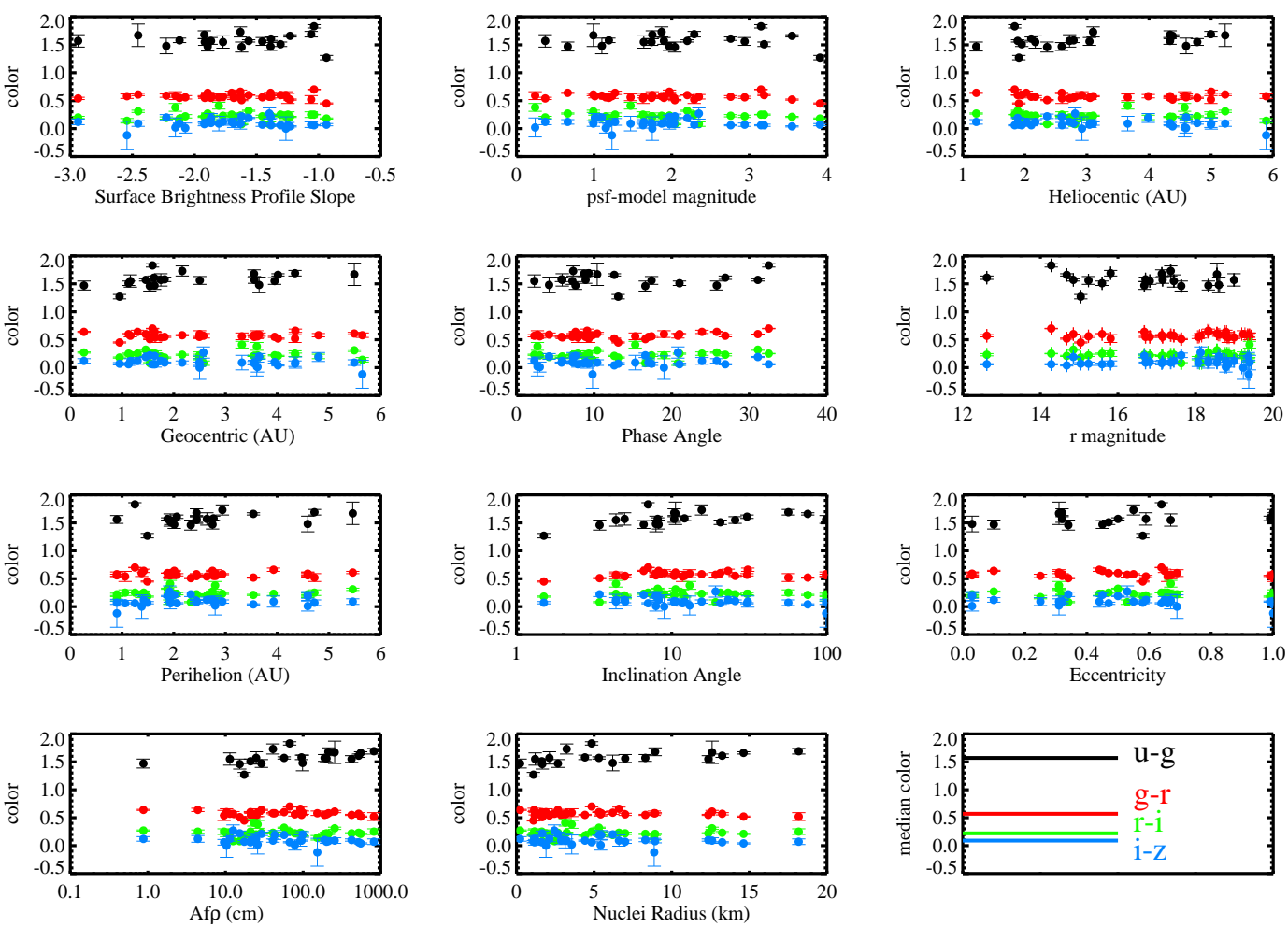

Fig. 6. The SDSS colors of comets are compared against various parameters, the lower right plot has a key to the colors in the plot, as well as showing the median value for each color. There is no statistically significant trend in color against any parameter. The slopes of these trends have been fit, and are presented in Fig 7 . 


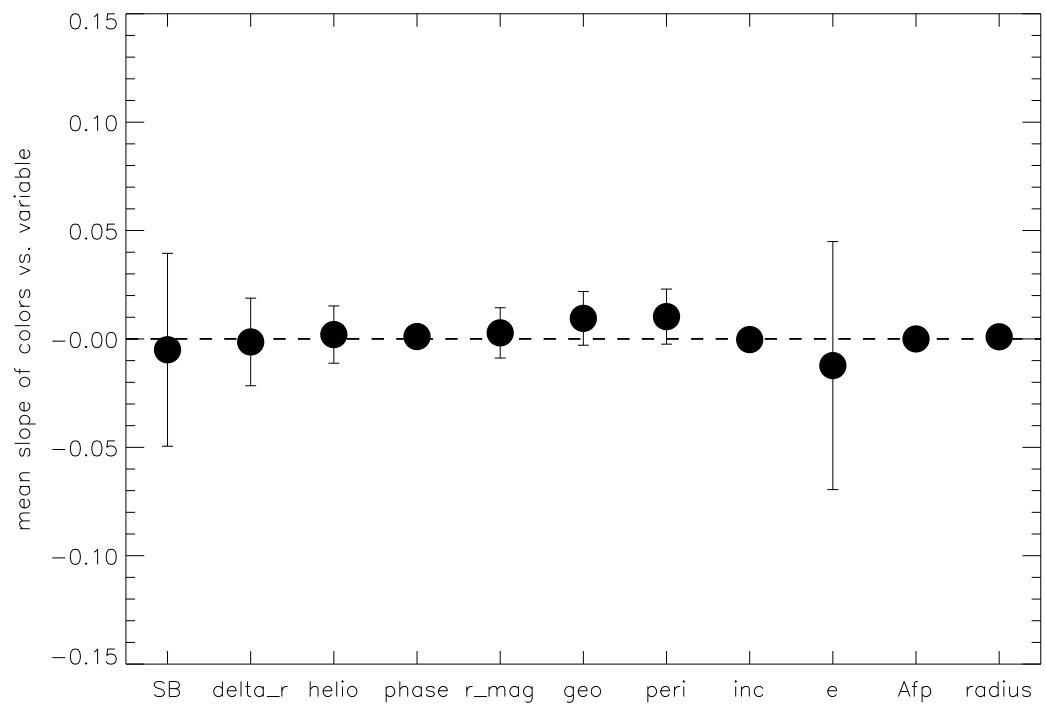

Fig. 7. The plot of mean color gradient of SDSS colors of comets against various parameters shows no color dependence on them. These same parameters can be seen in Figure 6. The abbreviations are:

-SB: Slope of the surface brightness profile

-delta_r: The $r$-band PSF-model magnitude

-helio: The heliocentric distance in AU at the time of observation

-phase: The phase angle at the time of observation

-r_mag: The $r$-band magnitude

-geo: The geocentric distance in $\mathrm{AU}$ at the time of observation

-peri: The perihelion distance in $\mathrm{AU}$

-inc: The orbital inclination

-e: The orbital eccentricity

-A $f \rho$ : The calculated A $f \rho$ value in $\mathrm{cm}$

-radius: The calculated radius limit in $\mathrm{km}$
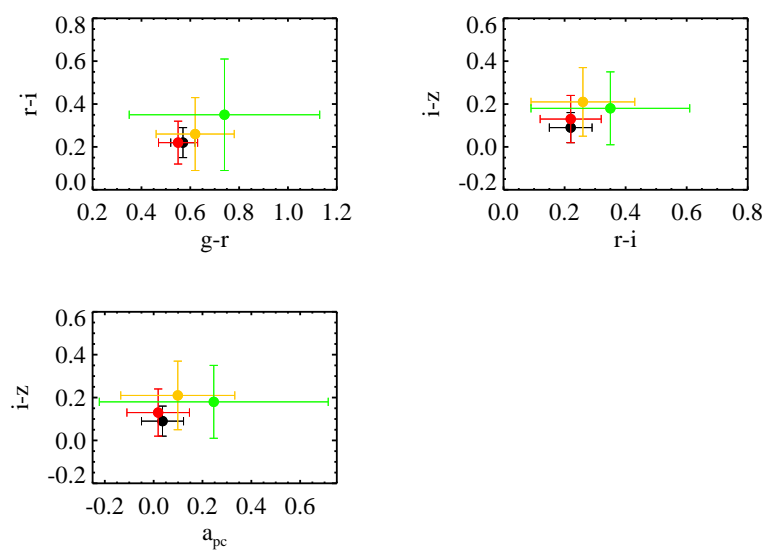

Fig. 8. Color-color diagrams of the various small body populations discussed in 4.1. The active comets are in black, the Trojans are in red, the Centaurs are in orange, and the TNO's are in green. The error bars represent one standard deviation. 

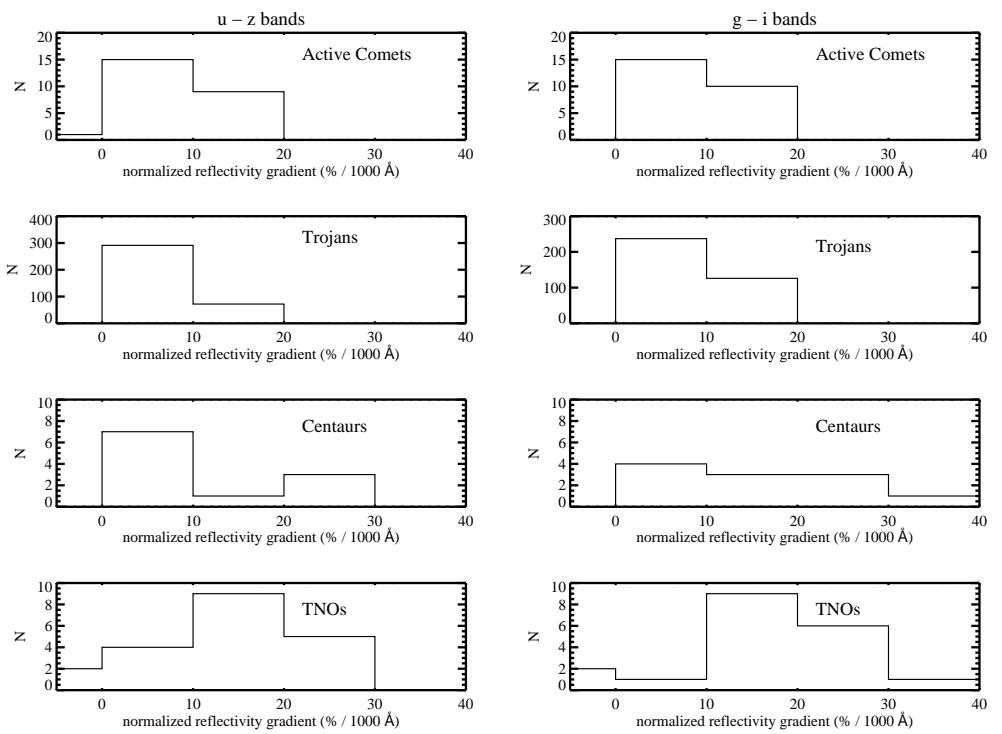

Fig. 9. Histograms of $S^{\prime}$ for the various small body populations discussed in 4.1.
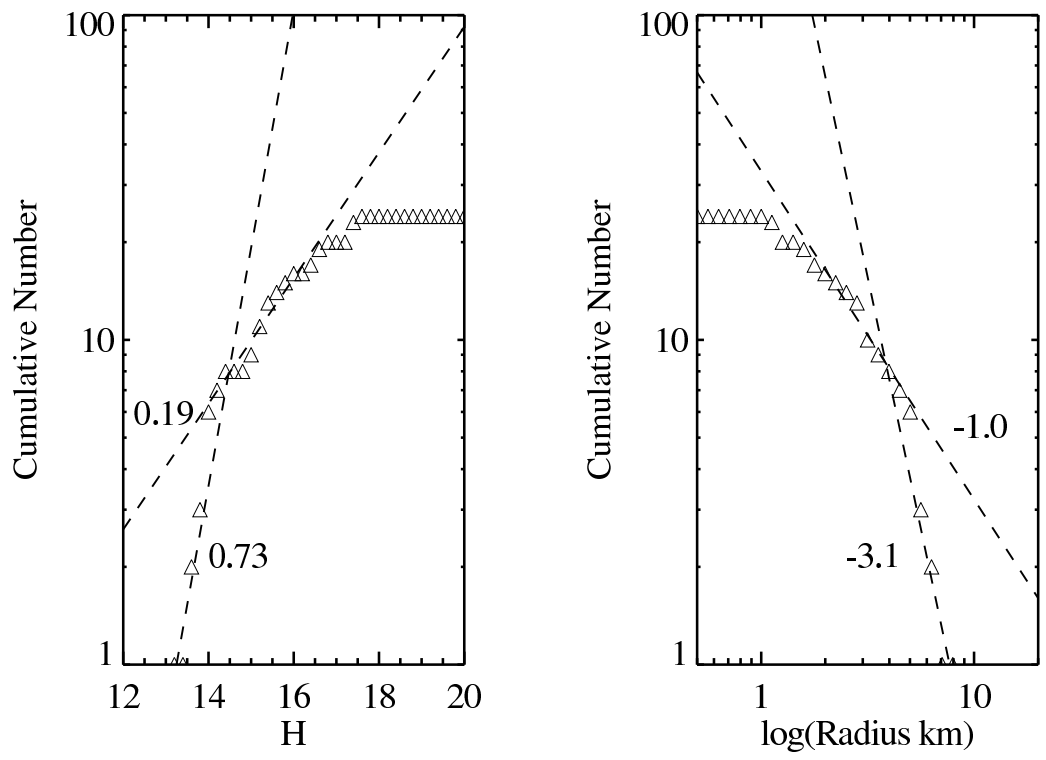

Fig. 10. Left: the Cumulative Luminosity Function for the Jupiter Family Comets in this paper, with power law fits. The whole population may be fit with a gradiant of $0.49 \pm 0.05$. Fitting with a broken power law at $H \sim 14.5$ gives gradients of $0.73 \pm 0.08$ and $0.19 \pm 0.03$ for the two fits, suggesting the faint population may be shallower than indicated by the single fit. Right: the Size Distribution Function for the same sample, assuming an albedo of 0.04. There is a break between the two measured slopes at $\sim 4 \mathrm{~km}$. The gradients are $-3.1 \pm 0.3$ and $-1.0 \pm 0.1$ for the broken fit. Fitting the whole range gives a fit of $-2.4 \pm 0.2$. 

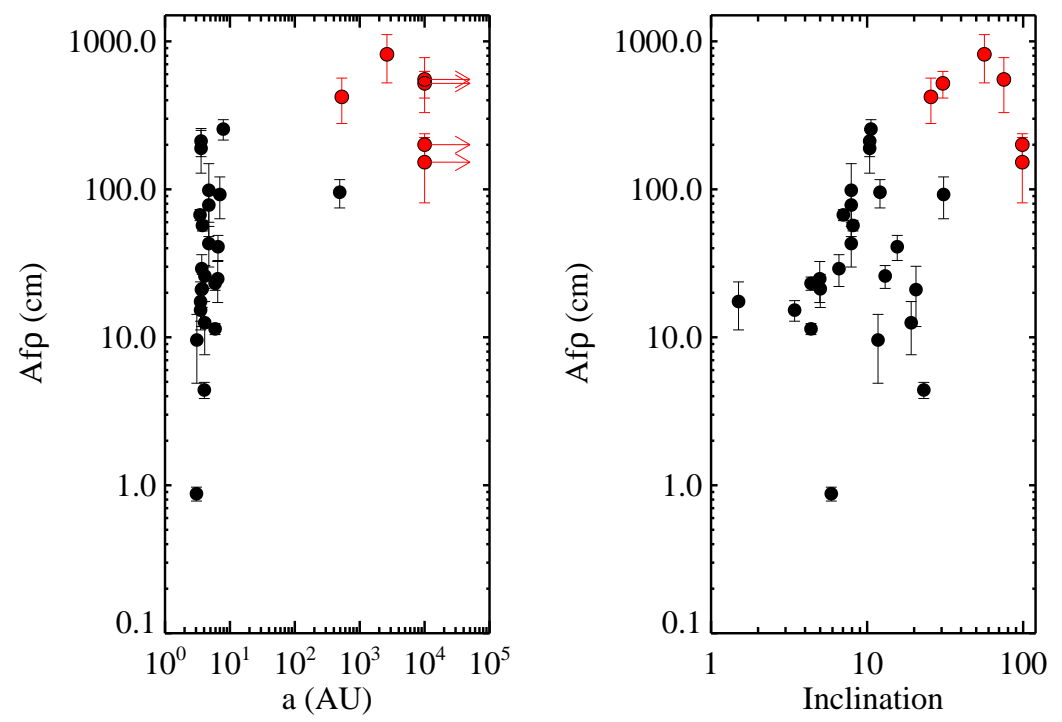

Fig. 11. Calculated Af $\rho$ values are compared on the left to the semi-major axis (a) and on the right to orbital inclination of the comets. Jupiter Family Comets are in black. The non-JFC population shows statistically larger Af $\rho$ values. Note: LPCs plotted at $10^{4} \mathrm{AU}$ are comets on unbound orbits, indicated by arrows. 